\title{
Spectral discretization of an unsteady flow through a porous solid
}

\author{
Christine Bernardi ${ }^{1}$, Sarra Maarouf ${ }^{1}$, and Driss Yakoubi ${ }^{2}$
}

\begin{abstract}
We consider the non stationary flow of a viscous incompressible fluid in a rigid homogeneous porous medium provided with mixed boundary conditions. Since the boundary pressure can present high variations, the permeability of the medium also depends on the pressure, so that the problem is nonlinear. We propose a discretization of this equation that combines Euler's implicit scheme in time and spectral methods in space. We prove optimal a priori error estimates and present some numerical experiments which confirm the interest of the discretization.
\end{abstract}

Résumé: Nous considérons l'écoulement instationnaire d'un fluide visqueux incompressible dans un milieu poreux rigide avec des conditions aux limites mixtes. Comme la pression sur la frontière peut présenter de fortes variations, la perméabilité du milieu est supposée dépendre de la pression de sorte que le modèle est non linéaire. Nous proposons une discrétisation en temps et en espace du système complet en utilisant le schéma d'Euler implicite et les méthodes spectrales. Nous prouvons des estimations d'erreur optimales et présentons quelques expériences numériques qui confirment l'intérêt de la discrétisation.

Key words: Darcy's equations, time discretization, spectral method.

\footnotetext{
${ }^{1}$ Laboratoire Jacques-Louis Lions, C.N.R.S. \& Université Pierre et Marie Curie, Boite courier 187, 4 place Jussieu, 75252 Paris cedex 05, France. e-mails: bernardi@ann.jussieu.fr,maarouf@ann.jussieu.fr

${ }^{2}$ GIREF, Département de Mathématiques et de Statistique, Pavillon Vachon, 1045 avenue de la médecine, Université de Laval, Québec, Canada. e-mail: yakoubi@giref.ulaval.ca
} 



\section{Introduction}

In this paper, we consider the nonlinear time-dependent Darcy's equations with mixed boundary conditions, first suggested by K.R. Rajagopal [16]. Let $\Omega$ be an open connected bounded domain in $\mathbb{R}^{d}, d=2$ or 3 , with a Lipschitz-continuous boundary $\partial \Omega$ divided in two parts $\Gamma_{u}$ and $\Gamma_{p}=\partial \Omega \backslash \bar{\Gamma}_{u}$ and $T$ be a positive real number. Let us introduce the unit outward normal vector to $\Omega$ on $\partial \Omega$ denoted by $\boldsymbol{n}$. For a given body force $\boldsymbol{f}$ (possibly depending on time) and a given initial velocity $\boldsymbol{u}_{0}$, the flow is assumed to be governed by the following boundary value problem

$$
\left\{\begin{array}{rll}
\partial_{t} \boldsymbol{u}+\alpha(p) \boldsymbol{u}+\nabla p=\boldsymbol{f} & \text { in } & \Omega \times] 0, T[, \\
\nabla \cdot \boldsymbol{u}=0 & \text { in } & \Omega \times] 0, T[, \\
p=p_{b} & \text { on } & \left.\Gamma_{p} \times\right] 0, T[, \\
\boldsymbol{u} \cdot \boldsymbol{n}=g & \text { on } & \left.\Gamma_{u} \times\right] 0, T[, \\
\left.\boldsymbol{u}\right|_{t=0}=\boldsymbol{u}_{0} & \text { on } & \Omega,
\end{array}\right.
$$

where the unknowns are the velocity $\boldsymbol{u}$ and pressure $p$ of the fluid. This system models the unsteady flow of an incompressible viscous fluid in a saturated rigid porous medium, in the case where the pressure $p$ presents high variations. Indeed, in this case, the coefficient $\alpha$ depends on these values in an exponential way. We refer to [16] for details on the way of deriving this model. Depending on the specific initial boundary value, problem under consideration has mixed boundary conditions where the pressure is described on a part of the boundary and the normal component of the velocity on the rest of the boundary.

It is well-known [8, Sec. XIII.1] that the steady linear Darcy's system has several equivalent variational formulations, according as the boundary conditions are treated as essential or natural ones. We choose one of these formulations which is more appropriate for the discretization and also for handling the time dependence, and we prove the existence of the solution of system (1.1). We refer to [4] for a first analysis of this nonlinear problem in the steady case.

We propose a time semi-discrete problem that relies on Euler's implicit scheme, however we have decided to treat the nonlinear term in an explicit way to make the implementation both simpler and less expensive. For the space discretization, we propose a spectral method in the basic situation where the domain is a square or a cube. More complex geometries can be treated thanks to the arguments in [14], however we prefer to avoid them for simplicity. The numerical analysis of the discrete problem makes use of the theory of F. Brezzi, J. Rappaz and P.-A. Raviart [11]. Thus, we establish a priori error estimates that turn out to be fully optimal. Finally, some numerical experiments confirm the interest of this approach.

The outline of the paper is as follows.

- In section 2, we present the variational formulation of problem (1.1) and prove that it is well-posed.

- Section 3 and 4 are devoted to the description of the time semi-discrete problem and of the fully discrete problem. We check their well-posedness.

- In Section 5, we assume that the permeability is constant and we describe the a priori analysis in this case.

- In section 6, we perform the a priori analysis of the discretization for problem (1.1) and prove optimal error estimates.

- Some numerical experiments are given in section 7 , these confirm the interest of the discretization. 


\section{The time-dependent Darcy's equations}

We first write a variational formulation of problem (1.1) and we prove the existence of the solution.

\subsection{Some preliminary notation}

From now on, we assume that,

(i) the intersection $\bar{\Gamma}_{u} \cap \bar{\Gamma}_{p}$ is a Lipschitz-continuous submanifold of $\partial \Omega$;

(ii) $\Gamma_{p}$ has a positive $(d-1)$-mesure in $\partial \Omega$;

(iii) the quantity $\alpha(p)$ is a Lipschitz-continuous function of the argument $p$, which is bounded from above and from below by positive constants $\alpha_{1}$ and $\alpha_{2}$

$$
\forall \xi \in \mathbb{R}, \quad \alpha_{1} \leq \alpha(\xi) \leq \alpha_{2} .
$$

In what follows, the scalar product defined on $L^{2}(\Omega)$ or $L^{2}(\Omega)^{d}$ is denoted by $(\cdot, \cdot)$. As usual, $H^{s}(\Omega), s \in \mathbb{R}$, denotes the real Sobolev space equipped with the norm $\|\cdot\|_{H^{s}(\Omega)}$ and semi-norm $|\cdot|_{H^{s}(\Omega)}$ (see for instance [2, Chap. III and VII]). For a fixed positive real number $T$ and a separable Banach space $E$ equipped with the norm $\|\cdot\|_{E}$, we denote by $\mathcal{C}^{0}(0, T ; E)$ the space of continuous functions from $[0, T]$ with values in $E$. For a nonnegative integer $s$, we also introduce the space $H^{s}(0, T ; E)$ in the following way: It is the space of measurable functions on ]0,T[ with values in $E$ such that the mappings: $v \mapsto\left\|\partial_{t}^{\ell} v\right\|_{E}, 0 \leq \ell \leq s$, are square-integrable on $] 0, T[$.

To write Darcy's problem (1.1) in variational form, we introduce the pressure space defined as follows:

$$
H_{(p)}^{1}(\Omega)=\left\{q \in H^{1}(\Omega) ; q=0 \text { on } \Gamma_{p}\right\} .
$$

The traces of functions in $H_{(p)}^{1}(\Omega)$ on $\Gamma_{u}$ belong to $H_{00}^{\frac{1}{2}}\left(\Gamma_{u}\right)$ (see $[13$, Chap.1, $\S 11]$ for the definition of this space). We introduce its dual space $H_{00}^{\frac{1}{2}}\left(\Gamma_{u}\right)^{\prime}$ and denote by $\langle\cdot, \cdot\rangle_{\Gamma_{u}}$ the duality pairing between $H_{00}^{\frac{1}{2}}\left(\Gamma_{u}\right)$ and $H_{00}^{\frac{1}{2}}\left(\Gamma_{u}\right)^{\prime}$. Thus, we assume that the partition of $\partial \Omega$ into $\Gamma_{u}$ and $\Gamma_{p}$ is sufficiently smooth for $\mathcal{D}\left(\Omega \cup \Gamma_{u}\right)$ to be dense in $H_{(p)}^{1}(\Omega)$ (sufficient conditions for this are given in [5] for instance).

We recall from Bernardi et al [8, Chap. XIII] and Achdou, Bernardi and Coquel [1], that Darcy's equations even for a constant function $\alpha(\cdot)$ admit several variational formulations. We have chosen here the formulation which enables us to treat the boundary on $p$ as an essential one and also seems the best adapted for handling the nonlinear term $\alpha(p) \boldsymbol{u}$.

\subsection{Variational formulation}

For a given data $\left(\boldsymbol{f}, g, p_{b}, \boldsymbol{u}_{0}\right)$ such that

$$
\begin{gathered}
\boldsymbol{f} \in L^{2}\left(0, T ; L^{2}(\Omega)^{d}\right), \quad g \in L^{2}\left(0, T ; H_{00}^{\frac{1}{2}}\left(\Gamma_{u}\right)^{\prime}\right), \\
p_{b} \in L^{2}\left(0, T ; H^{\frac{1}{2}}\left(\Gamma_{p}\right)\right) \quad \text { and } \quad \boldsymbol{u}_{0} \in L^{2}(\Omega)^{d},
\end{gathered}
$$


the weak formulation of problem (1.1) can be written as

$$
\begin{aligned}
& \text { Find }(\boldsymbol{u}, p) \in H^{1}\left(0, T ; L^{2}(\Omega)^{d}\right) \times L^{2}\left(0, T ; H^{1}(\Omega)\right) \text { such that } \\
& \qquad \boldsymbol{u}(\cdot, 0)=\boldsymbol{u}_{0} \text { in } \Omega
\end{aligned}
$$

and for a.e. $t \in] 0, T[$,

$$
\begin{aligned}
p(\cdot, t)=p_{b}(\cdot, t) \text { on } \Gamma_{p}, \\
\forall \boldsymbol{v} \in L^{2}(\Omega)^{d}, \quad \int_{\Omega} \partial_{t} \boldsymbol{u}(\boldsymbol{x}, t) \boldsymbol{v}(\boldsymbol{x}) d \boldsymbol{x}+\int_{\Omega}(\alpha(p) \boldsymbol{u})(\boldsymbol{x}, t) \boldsymbol{v}(\boldsymbol{x}) d \boldsymbol{x} \\
+b(\boldsymbol{v}, p)=\int_{\Omega} \boldsymbol{f}(\boldsymbol{x}, t) \cdot \boldsymbol{v}(\boldsymbol{x}) d \boldsymbol{x},
\end{aligned}
$$

$\forall q \in H_{(p)}^{1}(\Omega), \quad b(\boldsymbol{u}, q)=<g, q>_{\Gamma_{u}}$,

where the bilinear form $b(\cdot, \cdot)$ is defined by

$$
b(\boldsymbol{v}, q)=\int_{\Omega} \boldsymbol{v}(\boldsymbol{x}) \cdot \nabla q(\boldsymbol{x}) d \boldsymbol{x} .
$$

The following proposition states that system (1.1) is equivalent to the variational formulation (2.4) to (2.7). This is a consequence of usual density arguments.

Proposition 2.1 For any $\left(\boldsymbol{f}, g, p_{b}, \boldsymbol{u}_{0}\right)$ satisfying (2.3) problems (1.1) and (2.4) to (2.7) are equivalent in the sense that the pair $(\boldsymbol{u}, p)$ in $H^{1}\left(0, T ; L^{2}(\Omega)^{d}\right) \times L^{2}\left(0, T ; H^{1}(\Omega)\right)$ is a solution of (1.1) in the distribution sense if and only if it is a solution of problem (2.4) to (2.7).

\subsection{Existence result}

We are now in a position to derive the main result of this section, namely the existence of a solution to problem (2.4) to (2.7).

First, it is clear that the bilinear form $b(\cdot, \cdot)$ is continuous on $L^{2}(\Omega)^{d} \times H^{1}(\Omega)$ and satisfies the following inf-sup condition (the proof consists in taking $\boldsymbol{v}$ equal to $\nabla q$ )

$$
\forall q \in H^{1}(\Omega), \quad \sup _{\boldsymbol{v} \in L^{2}(\Omega)^{d}} \frac{b(\boldsymbol{v}, q)}{\|\boldsymbol{v}\|_{L^{2}(\Omega)^{d}}} \geq|q|_{H^{1}(\Omega)} .
$$

Furthermore, its kernel

$$
\mathbf{V}(\Omega)=\left\{\boldsymbol{v} \in L^{2}(\Omega)^{d} ; \quad \forall q \in H_{(p)}^{1}(\Omega), \quad b(\boldsymbol{v}, q)=0\right\}
$$

can be characterized by

$$
\mathbf{V}(\Omega)=\left\{\boldsymbol{v} \in L^{2}(\Omega)^{d} ; \quad \nabla \cdot \boldsymbol{v}=0 \text { in } \Omega \text { and } \boldsymbol{v} \cdot \boldsymbol{n}=0 \text { on } \Gamma_{u}\right\} .
$$

Thus, this space is separable (see [9, Prop. III.22]). The importance of this space appears later on.

Thanks to the assumption $p_{b} \in L^{2}\left(0, T ; H^{\frac{1}{2}}\left(\Gamma_{p}\right)\right)$, there exists a lifting still denoted by $p_{b}$ for simplicity, which belongs to $L^{2}\left(0, T ; H^{1}(\Omega)\right)$ such that

$$
\left\|p_{b}\right\|_{L^{2}\left(0, T ; H^{1}(\Omega)\right)} \leq c_{0}\left\|p_{b}\right\|_{L^{2}\left(0, T ; H^{\frac{1}{2}}\left(\Gamma_{p}\right)\right)}
$$

where the nonnegative constant $c_{0}$ only depends on $\Omega$. On the other hand, due to the inf-sup condition $(2.9)$ and the continuity of the trace operator from $H_{(p)}^{1}(\Omega)$ into $H_{00}^{\frac{1}{2}}\left(\Gamma_{u}\right)$, there exists 
an isomorphism $B$ from $H_{00}^{\frac{1}{2}}\left(\Gamma_{u}\right)^{\prime}$ into the orthogonal of $\mathbf{V}(\Omega)$, (see Girault-Raviart [12, Chap. I, Lem. 4.1]), such that, when setting $\boldsymbol{w}(\cdot, t)=B g(\cdot, t)$, for a.e. $t$ in $[0, T]$ (recall that the datum $g$ belongs to $\left.L^{2}\left(0, T ; H_{00}^{\frac{1}{2}}\left(\Gamma_{u}\right)^{\prime}\right)\right)$

$$
\forall q \in H_{(p)}^{1}(\Omega), \quad b(\boldsymbol{w}(\cdot, t), q)=<g(\cdot, t), q>_{\Gamma_{u}},
$$

and

$$
\|\boldsymbol{w}(\cdot, t)\|_{L^{2}(\Omega)^{d}} \leq\|g(\cdot, t)\|_{H_{00}^{\frac{1}{2}}\left(\Gamma_{u}\right)^{\prime}}
$$

Let us introduce the mapping $\alpha_{*}$,

$$
\forall(\boldsymbol{x}, t) \in \Omega \times] 0, T\left[, \quad \forall \xi \in \mathbb{R}, \quad \alpha_{*}(\boldsymbol{x}, t, \xi)=\alpha\left(\xi+p_{b}(\boldsymbol{x}, t)\right) .\right.
$$

Obviously it satisfies the same properties (2.1) as $\alpha$. For brevity, in what follows $\alpha_{*}(\xi)$ stands for $\alpha_{*}(\boldsymbol{x}, t, \xi)$.

Next, we set $\boldsymbol{u}=\boldsymbol{u}_{*}+\boldsymbol{w}$ and $p=p_{*}+p_{b}$, we observe that the pair $\left(\boldsymbol{u}_{*}, p_{*}\right)$ is a solution of the following variational problem

$$
\begin{aligned}
\text { Find }\left(\boldsymbol{u}_{*}, p_{*}\right) \in H^{1}(0, T ; \mathbf{V}(\Omega)) & \times L^{2}\left(0, T ; H_{(p)}^{1}(\Omega)\right) \text { such that } \\
\boldsymbol{u}_{*}(\cdot, 0) & =\boldsymbol{u}_{0}-\boldsymbol{w}(\cdot, 0) \quad \text { in } \Omega,
\end{aligned}
$$

and

$$
\begin{aligned}
\forall \boldsymbol{v} \in \mathbf{V}(\Omega), \quad \int_{\Omega} \partial_{t} \boldsymbol{u}_{*}(\boldsymbol{x}, t) \boldsymbol{v}(\boldsymbol{x}) d \boldsymbol{x}+\int_{\Omega} \alpha_{*}\left(p_{*}(\boldsymbol{x}, t)\right) \boldsymbol{u}_{*}(\boldsymbol{x}, t) \boldsymbol{v}(\boldsymbol{x}) d \boldsymbol{x} \\
\quad=\int_{\Omega} \boldsymbol{f}(\boldsymbol{x}, t) \cdot \boldsymbol{v}(\boldsymbol{x}) d \boldsymbol{x}-\int_{\Omega} \alpha_{*}\left(p_{*}(\boldsymbol{x}, t)\right) \boldsymbol{w}(\boldsymbol{x}, t) \boldsymbol{v}(\boldsymbol{x}) d \boldsymbol{x}-b\left(\boldsymbol{v}, p_{b}\right) .
\end{aligned}
$$

The following equivalence property is readily checked, see [6, Lem. 2.1].

Proposition 2.2 The variational problems (2.4) to (2.7) and (2.13)-(2.14) are equivalent in the sense that $\left(\boldsymbol{u}_{*}, p_{*}\right)$ is solution of (2.13)-(2.14) if and only if $(\boldsymbol{u}, p)$ is solution of (2.4) to $(2.7)$.

To go further, we prove a stability property of the solution $\boldsymbol{u}_{*}$.

Lemma 2.3 For any data $\left(\boldsymbol{f}, g, p_{b}, \boldsymbol{u}_{0}\right)$ satisfying (2.3), the following estimates hold for any solution $\boldsymbol{u}_{*}$ of problem (2.13)-(2.14) and for any $t$ in $[0, T]$

$$
\begin{aligned}
& \left\|\boldsymbol{u}_{*}(\cdot, t)\right\|_{L^{2}(\Omega)^{d}}^{2}+\alpha_{1}\left\|\boldsymbol{u}_{*}\right\|_{L^{2}\left(0, t, L^{2}(\Omega)^{d}\right)}^{2} \\
& \quad \leq c\left(\left\|\boldsymbol{u}_{*}(\cdot, 0)\right\|_{L^{2}(\Omega)^{d}}^{2}+\|\boldsymbol{f}\|_{L^{2}\left(0, t ; L^{2}(\Omega)^{d}\right)}^{2}+\left\|p_{b}\right\|_{L^{2}\left(0, t ; H^{\left.\frac{1}{2}\left(\Gamma_{p}\right)\right)}\right.}^{2}+\|g\|_{L^{2}\left(0, t ; H_{00}\right.}^{\left.\frac{1}{2}\left(\Gamma_{u}\right)^{\prime}\right)}\right) .
\end{aligned}
$$

and

$$
\begin{aligned}
& \left\|\partial_{t} \boldsymbol{u}_{*}\right\|_{L^{2}\left(0, t ; L^{2}(\Omega)^{d}\right)}^{2} \\
& \quad \leq c\left(\left\|\boldsymbol{u}_{*}(\cdot, 0)\right\|_{L^{2}(\Omega)^{d}}^{2}+\|\boldsymbol{f}\|_{L^{2}\left(0, t ; L^{2}(\Omega)^{d}\right)}^{2}+\left\|p_{b}\right\|_{L^{2}\left(0, t ; H^{\left.\frac{1}{2}\left(\Gamma_{p}\right)\right)}\right.}^{2}+\|g\|_{L^{2}\left(0, t ; H_{00}\right.}^{\left.\frac{1}{2}\left(\Gamma_{u}\right)^{\prime}\right)}\right) .
\end{aligned}
$$


Proof. Taking $\boldsymbol{v}=\boldsymbol{u}_{*}$ in (2.14), and using the Cauchy-Schwarz inequality and hypothesis (2.1) we obtain

$$
\begin{aligned}
\frac{1}{2} \int_{\Omega}\left(\frac{d \boldsymbol{u}_{*}^{2}}{d t}\right)(\boldsymbol{x}, t) d \boldsymbol{x} & +\frac{\alpha_{1}}{2}\left\|\boldsymbol{u}_{*}(\cdot, t)\right\|_{L^{2}(\Omega)^{d}}^{2} \\
& \leq \frac{3}{2 \alpha_{1}}\left(\|\boldsymbol{f}(\cdot, t)\|_{L^{2}(\Omega)^{d}}^{2}+\left|p_{b}(\cdot, t)\right|_{H^{1}(\Omega)}^{2}\right)+\frac{3 \alpha_{2}^{2}}{2 \alpha_{1}}\|\boldsymbol{w}(\cdot, t)\|_{L^{2}(\Omega)^{d}}^{2},
\end{aligned}
$$

whence, by integrating with respect to $t$,

$$
\begin{aligned}
& \left\|\boldsymbol{u}_{*}(\cdot, t)\right\|_{L^{2}(\Omega)^{d}}^{2}+\alpha_{1}\left\|\boldsymbol{u}_{*}\right\|_{L^{2}\left(0, t ; L^{2}(\Omega)^{d}\right)}^{2} \\
& \quad \leq\left\|\boldsymbol{u}_{*}(\cdot, 0)\right\|_{L^{2}(\Omega)^{d}}^{2}+\frac{3}{\alpha_{1}}\left(\|\boldsymbol{f}\|_{L^{2}\left(0, t ; L^{2}(\Omega)^{d}\right)}^{2}+\left\|p_{b}\right\|_{L^{2}\left(0, t ; H^{1}(\Omega)\right)}^{2}\right)+\frac{3 \alpha_{2}^{2}}{\alpha_{1}}\|\boldsymbol{w}\|_{L^{2}\left(0, t ; L^{2}(\Omega)^{d}\right)^{2}}^{2} .
\end{aligned}
$$

When combined with (2.11) and (2.12), this yields the desired estimate (2.15). To prove the second estimate (2.16), we take $\boldsymbol{v}=\partial_{t} \boldsymbol{u}_{*}$ in (2.14),

$$
\begin{aligned}
& \left\|\partial_{t} \boldsymbol{u}_{*}(\cdot, t)\right\|_{L^{2}(\Omega)^{d}}^{2}+\int_{\Omega} \alpha_{*}\left(p_{*}(\boldsymbol{x}, t)\right) \boldsymbol{u}_{*}(\boldsymbol{x}, t) \partial_{t} \boldsymbol{u}_{*}(\boldsymbol{x}, t) d \boldsymbol{x} \\
& =\int_{\Omega} \boldsymbol{f}(\boldsymbol{x}, t) \partial_{t} \boldsymbol{u}_{*}(\boldsymbol{x}, t) d \boldsymbol{x}-\int_{\Omega} \alpha_{*}\left(p_{*}(\boldsymbol{x}, t)\right) \boldsymbol{w}(\boldsymbol{x}, t) \partial_{t} \boldsymbol{u}_{*}(\boldsymbol{x}, t) d \boldsymbol{x}-b\left(\partial_{t} \boldsymbol{u}_{*}, p_{b}\right) .
\end{aligned}
$$

Thus,

$$
\begin{aligned}
\left\|\partial_{t} \boldsymbol{u}_{*}(\cdot, t)\right\|_{L^{2}(\Omega)^{d}}^{2} & \\
& \leq\|\boldsymbol{f}(\cdot, t)\|_{L^{2}(\Omega)^{d}}\left\|\partial_{t} \boldsymbol{u}_{*}(\cdot, t)\right\|_{L^{2}(\Omega)^{d}}+\left|p_{b}(\cdot, t)\right|_{H^{1}(\Omega)}\left\|\partial_{t} \boldsymbol{u}_{*}(\cdot, t)\right\|_{L^{2}(\Omega)^{d}} \\
& +\alpha_{2}\|\boldsymbol{w}(\cdot, t)\|_{L^{2}(\Omega)^{d}}\left\|\partial_{t} \boldsymbol{u}_{*}(\cdot, t)\right\|_{L^{2}(\Omega)^{d}}+\alpha_{2}\left\|\boldsymbol{u}_{*}(\cdot, t)\right\|_{L^{2}(\Omega)^{d}}\left\|\partial_{t} \boldsymbol{u}_{*}(\cdot, t)\right\|_{L^{2}(\Omega)^{d}},
\end{aligned}
$$

and we use the same arguments as previously and (2.15).

As a consequence of the definition of $\boldsymbol{u}_{*}$, the previous Lemma 2.3 and a triangle inequality imply the following estimate for the velocity.

Corollary 2.4 For any data

$$
\begin{gathered}
\boldsymbol{f} \in L^{2}\left(0, T ; L^{2}(\Omega)^{d}\right), \quad g \in H^{1}\left(0, T ; H_{00}^{\frac{1}{2}}\left(\Gamma_{u}\right)^{\prime}\right), \\
p_{b} \in L^{2}\left(0, T ; H^{\frac{1}{2}}\left(\Gamma_{p}\right)\right) \quad \text { and } \quad \boldsymbol{u}_{0} \in L^{2}(\Omega)^{d},
\end{gathered}
$$

the solution $\boldsymbol{u}$ of problem (2.4) to (2.7) satisfies for all $0 \leq t \leq T$,

$$
\begin{aligned}
\|\boldsymbol{u}\|_{L^{\infty}\left(0, T ; L^{2}(\Omega)^{d}\right)} & \leq c^{\prime}\left(\left\|\boldsymbol{u}_{0}\right\|_{L^{2}(\Omega)^{d}}+\|\boldsymbol{f}\|_{L^{2}\left(0, T ; L^{2}(\Omega)^{d}\right)}\right. \\
& \left.+\left\|p_{b}\right\|_{L^{2}\left(0, T ; H^{\frac{1}{2}}\left(\Gamma_{p}\right)\right)}+\sup _{0 \leq t \leq T}\|g(\cdot, t)\|_{H_{00}^{\frac{1}{2}\left(\Gamma_{u}\right)^{\prime}}}+\|g\|_{L^{2}\left(0, T ; H_{00}\left(\Gamma_{u}\right)^{\prime}\right)}\right) .
\end{aligned}
$$

It follows from the definition $(2.10)$ of $\mathbf{V}(\Omega)$ that $L^{2}(\Omega)^{d}$ is the orthogonal sum of $\mathbf{V}(\Omega)$ and $\nabla H_{(p)}^{1}(\Omega)$. So problem $(2.4)$ to $(2.7)$ can be rewritten as the sum of two coupled equations

Find $\left(\boldsymbol{u}_{*}, p_{*}\right) \in H^{1}(0, T ; \mathbf{V}(\Omega)) \times L^{2}\left(0, T ; H_{(p)}^{1}(\Omega)\right)$ satisfying $(2.13)$, such that

$$
\begin{aligned}
& \forall \boldsymbol{v} \in \mathbf{V}(\Omega), \quad\left(\partial_{t} \boldsymbol{u}_{*}, \boldsymbol{v}\right)+\left(\alpha_{*}\left(p_{*}\right) \boldsymbol{u}_{*}, \boldsymbol{v}\right)+\left(\alpha_{*}\left(p_{*}\right) \boldsymbol{w}, \boldsymbol{v}\right)=\left(\boldsymbol{f}_{*}, \boldsymbol{v}\right), \\
& \forall q \in H_{(p)}^{1}(\Omega), \quad\left(\alpha_{*}\left(p_{*}\right) \boldsymbol{u}_{*}, \nabla q\right)+\left(\alpha_{*}\left(p_{*}\right) \boldsymbol{w}, \nabla q\right)+b\left(\nabla q, p_{*}\right)=\left(\boldsymbol{f}_{*}, \nabla q\right),
\end{aligned}
$$

where the new function $\boldsymbol{f}_{*}$ is equal to $\boldsymbol{f}-\partial_{t} \boldsymbol{w}-\nabla p_{b}$.

We refer to [6, Thm. 2.4], for the detailed proof of the next result, since it is a direct consequence of the Cauchy-Lipschitz theorem and the separability of $\mathbf{V}(\Omega)$. 
Proposition 2.5 For any data $\left(\boldsymbol{f}, g, p_{b}, \boldsymbol{u}_{0}\right)$ satisfying $(2.3)$ and for any $p_{*}$ in $L^{2}\left(0, T ; L^{2}(\Omega)\right)$, problem (2.18) has a unique solution $\boldsymbol{u}_{*}$ in $L^{2}(0, T ; \mathbf{V}(\Omega))$ which satisfies (2.15) and (2.16).

We introduce the mapping $\mathcal{F}: p_{*} \mapsto \boldsymbol{u}_{*}$ where $\boldsymbol{u}_{*}$ is the solution given in the last proposition. This mapping is continuous from $L^{2}\left(0, T ; L^{2}(\Omega)\right)$ into $\mathcal{C}^{0}(0, T ; \mathbf{V}(\Omega))$ and thanks to (2.15), it is bounded.

Proposition 2.6 For any data $\left(\boldsymbol{f}, g, p_{b}, \boldsymbol{u}_{0}\right)$ satisfying (2.17), problem (2.19) has a solution $p_{*}$ in $L^{2}\left(0, T ; H_{(p)}^{1}(\Omega)\right)$. Moreover this solution satisfies

$$
\begin{aligned}
\left|p_{*}\right|_{L^{2}\left(0, T ; H^{1}(\Omega)\right)} \leq \quad c\left(\|\boldsymbol{f}\|_{L^{2}\left(0, T ; L^{2}(\Omega)^{d}\right)}+\left\|p_{b}\right\|_{L^{2}\left(0, T ; H^{\frac{1}{2}}\left(\Gamma_{p}\right)\right)}\right. \\
\left.+\|g\|_{H^{1}\left(0, T ; H_{00}^{\frac{1}{2}}\left(\Gamma_{u}\right)^{\prime}\right)}+\left\|\boldsymbol{u}_{0}\right\|_{L^{2}(\Omega)^{d}}\right) .
\end{aligned}
$$

Proof. Since problem (2.19) is steady, we fix a time $t \in] 0, T$. For simplicity, we omit the $t$ in what follows. We define the mapping $\phi$, for any $q \in H_{(p)}^{1}(\Omega)$

$$
<\phi\left(p_{*}\right), q>=\left(\alpha_{*}\left(p_{*}\right) \mathcal{F}\left(p_{*}\right), \nabla q\right)+\left(\alpha_{*}\left(p_{*}\right) \boldsymbol{w}, \nabla q\right)+b\left(\nabla q, p_{*}\right)-\left(\boldsymbol{f}_{*}, \nabla q\right) .
$$

This mapping is continuous and satisfies by taking $q=p_{*}$,

$$
<\phi\left(p_{*}\right), p_{*}>\geq\left|p_{*}\right|_{H^{1}(\Omega)}^{2}-c_{0}(t)\left|p_{*}\right|_{H^{1}(\Omega)},
$$

where

$$
c_{0}(t)=\left\|\partial_{t} \boldsymbol{w}\right\|_{L^{2}(\Omega)^{d}}+\alpha_{2}\|\boldsymbol{w}\|_{L^{2}(\Omega)^{d}}+\|\boldsymbol{f}\|_{L^{2}(\Omega)^{d}}+\left|p_{b}\right|_{H^{1}(\Omega)}+\left\|\mathcal{F}\left(p_{*}\right)\right\|_{L^{2}(\Omega)^{d}},
$$

whence, from (2.15),

$$
c_{0}(t) \leq c\left(\|\boldsymbol{f}\|_{L^{2}\left(0, T ; L^{2}(\Omega)^{d}\right)}+\left\|p_{b}\right\|_{L^{2}\left(0, T ; H^{\frac{1}{2}}\left(\Gamma_{p}\right)\right)}+\|g\|_{H^{1}\left(0, T ; H_{00}^{\left.\frac{1}{2}\left(\Gamma_{u}\right)^{\prime}\right)}\right.}+\left\|\boldsymbol{u}_{0}\right\|_{L^{2}(\Omega)^{d}}\right) .
$$

Thus, the quantity $<\phi\left(p_{*}\right), p_{*}>$ is nonnegative on the sphere with radius $c_{0}(t)$. It follows from the density assumption that there exists an increasing sequence $\left(\mathbb{W}_{n}\right)_{n}$ of finite dimensional subspaces of $H_{(p)}^{1}(\Omega)$ such that $H_{(p)}^{1}(\Omega)=\cup_{n \in \mathbb{N}} \mathbb{W}_{n}$. For each $n$, the restriction of the mapping $\phi$ to each $\mathbb{W}_{n}$ obviously satisfies the same properties as previously, so that applying Brouwer's fixed point theorem (see [12, Chap. IV, Corollary 1.1] for instance) yields the existence of a function $p_{* n}$ in $\mathbb{W}_{n}$ such that

$$
\left|p_{*_{n}}\right|_{H^{1}(\Omega)} \leq c_{0}(t) \quad \text { and } \quad<\phi\left(p_{*_{n}}\right), q_{n}>=0, \quad \forall q_{n} \in \mathbb{W}_{n}
$$

Since $\left(p_{* n}\right)_{n}$ is bounded in $H_{(p)}^{1}(\Omega)$ there exists a subsequence still denoted by $\left(p_{* n}\right)_{n}$ which converges to a function $p_{*}$ weakly in $H^{1}(\Omega)$ and strongly in $L^{2}(\Omega)$. Owing to Poincaré-Fridrichs inequality, the seminorm $|\cdot|_{H^{1}(\Omega)}$ is a norm on $H_{(p)}^{1}(\Omega)$. So, it thus follows from the weak lower semi-continuity of the norm that

$$
\left|p_{*}(t)\right|_{H^{1}(\Omega)} \leq c_{0}(t)
$$

The function $p_{* n}$ satisfies for all $q_{m}$ in $\mathbb{W}_{m}, m \leq n$,

$$
\left(\alpha_{*}\left(p_{* n}\right) \mathcal{F}\left(p_{* n}\right), \nabla q_{m}\right)+\left(\alpha_{*}\left(p_{* n}\right) \boldsymbol{w}, \nabla q_{m}\right)+b\left(\nabla q_{m}, p_{* n}\right)=\left(\boldsymbol{f}_{*}, \nabla q_{m}\right) .
$$

Thanks the continuity of the mapping $\mathcal{F}$ on $L^{2}(\Omega)$, the sequence $\left(\mathcal{F}\left(p_{* n}\right)\right)_{n}$ converges to $\mathcal{F}\left(p_{*}\right)$ strongly in $L^{2}(\Omega)$. On the other hand, the sequence $\left(\alpha_{*}\left(p_{* n}\right) \nabla q_{m}\right)_{n}$ converges to $\alpha_{*}\left(p_{*}\right) \nabla q_{m}$ a.e. in $\Omega \times] 0, T$, and is bounded by $\alpha_{2} \nabla q_{m}$. Applying the Lebesgue dominated convergence theorem 
implies that $\alpha_{*}\left(p_{* n}\right) \nabla q_{m}$ converges to $\alpha_{*}\left(p_{*}\right) \nabla q_{m}$ in $L^{2}(\Omega)^{d}$. By combining these convergence properties and setting $u_{*}=\mathcal{F}\left(p_{*}\right)$, we observe that $p_{*}$ satisfies for all $q_{m}$ in $\mathbb{W}_{m}$,

$$
\left(\alpha_{*}\left(p_{*}\right) u_{*}, \nabla q_{m}\right)+\left(\alpha_{*}\left(p_{*}\right) \boldsymbol{w}, \nabla q_{m}\right)+b\left(\nabla q_{m}, p_{*}\right)=\left(\boldsymbol{f}_{*}, \nabla q_{m}\right) .
$$

Finally, the density of $\cup_{n \in \mathbb{N}} \mathbb{W}_{n}$ in $H_{(p)}^{1}(\Omega)$ implies that $p_{*}$ is a solution of problem (2.19). Estimate (2.20) is derived by combining (2.23) with (2.21) and integrating with respect to $t$.

To conclude the pair $\left(\boldsymbol{u}_{*}, p_{*}\right)$ is a solution of problem $(2.18)-(2.19)$.

All this leads to the next corollary.

Corollary 2.7 For any data $\left(\boldsymbol{f}, g, p_{b}, \boldsymbol{u}_{0}\right)$ satisfying (2.17), problem (2.4) to (2.7) has a solution $(\boldsymbol{u}, p)$ in $H^{1}\left(0, T ; L^{2}(\Omega)^{d}\right) \times L^{2}\left(0, T ; H^{1}(\Omega)\right)$. Moreover this solution satisfies

$$
\begin{aligned}
& \|\boldsymbol{u}\|_{L^{\infty}\left(0, T ; L^{2}(\Omega)^{d}\right)}+\|p\|_{L^{2}\left(0, T ; H^{1}(\Omega)\right)} \\
& \quad \leq c\left(\left\|\boldsymbol{u}_{0}\right\|_{L^{2}(\Omega)^{d}}+\|\boldsymbol{f}\|_{L^{2}\left(0, T ; L^{2}(\Omega)^{d}\right)}+\left\|p_{b}\right\|_{L^{2}\left(0, T ; H^{\left.\frac{1}{2}\left(\Gamma_{p}\right)\right)}\right.}+\|g\|_{H^{1}\left(0, T ; H_{00}^{\left.\frac{1}{2}\left(\Gamma_{u}\right)^{\prime}\right)}\right.}\right) .
\end{aligned}
$$

The uniqueness of the solution requires some rather restrictive conditions on the coefficient $\alpha$, so we prefer to skip its study.

\section{The time semi-discrete problem}

In order to approximate the solution of problem (1.1), we first propose a time semi-discretization. For that, we introduce a partition of the interval $[0, T]$ into subintervals $\left[t_{n-1}, t_{n}\right], 1 \leq n \leq N$, such that $0=t_{0}<t_{1}<\cdots<t_{N}=T$. We denote by $\tau_{n}$ the time step $t_{n}-t_{n-1}$, by $\tau$ the $N$-tuple $\left(\tau_{1}, \ldots, \tau_{N}\right)$ and by $|\tau|$ the maximum of the $\tau_{n}, 1 \leq n \leq N$. We assume that

$$
\max _{2 \leq n \leq N} \frac{\tau_{n}}{\tau_{n-1}} \leq \sigma
$$

where the constant $\sigma$ is independent of $\tau$. We denote by $\boldsymbol{u}^{n}$ and $p^{n}$ the approximate solution at time $t_{n}$. For the discretization of the time derivative, we use an implicit backward Euler's scheme. Nevertheless, for the sake of simplicity and clarity, we shall present our analysis by approximating the nonlinear term by $\alpha\left(p^{n-1}\right)$. We also suppose that the data $\boldsymbol{f}, g$ and $p_{b}$ are continuous in time.

In what follows, we introduce the initial pressure $p^{0}$ as a fixed function in $H^{1}(\Omega)$, for instance the atmospheric pressure. Then, the semi-discrete problem consists in

$$
\begin{aligned}
& \text { Find }\left(\boldsymbol{u}^{n}, p^{n}\right) \in L^{2}(\Omega)^{d} \times H^{1}(\Omega) \text { such that } \\
& \boldsymbol{u}^{0}=\boldsymbol{u}_{0} \quad \text { in } \Omega \text {, } \\
& \text { and for } n \geq 1 \text {, } \\
& p^{n}=p_{b}^{n} \quad \text { on } \Gamma_{p}, \\
& \forall \boldsymbol{v} \in L^{2}(\Omega)^{d}, \quad\left(\frac{\boldsymbol{u}^{n}-\boldsymbol{u}^{n-1}}{\tau_{n}}, \boldsymbol{v}\right)+\left(\alpha\left(p^{n-1}\right) \boldsymbol{u}^{n}, \boldsymbol{v}\right)+b\left(\boldsymbol{v}, p^{n}\right)=\left(\boldsymbol{f}^{n}, \boldsymbol{v}\right), \\
& \forall q \in H_{(p)}^{1}(\Omega), \quad b\left(\boldsymbol{u}^{n}, q\right)=\stackrel{\tau_{n}}{<} g^{n}, q>_{\Gamma_{u}},
\end{aligned}
$$

where $\boldsymbol{f}^{n}=\boldsymbol{f}\left(\cdot, t_{n}\right), g^{n}=g\left(\cdot, t_{n}\right)$ and $p_{b}^{n}=p_{b}\left(\cdot, t_{n}\right)$. We are now in position to establish the well-posedeness of this problem.

Proposition 3.1 If

$$
\begin{aligned}
& \boldsymbol{f} \in \mathcal{C}^{0}\left(0, T ; L^{2}(\Omega)^{d}\right), \quad g \in \mathcal{C}^{0}\left(0, T ; H_{00}^{\frac{1}{2}}\left(\Gamma_{u}\right)^{\prime}\right), \\
& p_{b} \in \mathcal{C}^{0}\left(0, T ; H^{\frac{1}{2}}\left(\Gamma_{p}\right)\right) \quad \text { and } \quad \boldsymbol{u}_{0} \in L^{2}(\Omega)^{d},
\end{aligned}
$$


problem (3.1) has a unique solution. Furthermore, this solution satisfies the following stability properties for $1 \leq n \leq N$

$$
\begin{aligned}
\left\|\boldsymbol{u}^{n}\right\|_{L^{2}(\Omega)^{d}} & \leq\left\|\boldsymbol{u}^{0}\right\|_{L^{2}(\Omega)^{d}}+\left\|g^{0}\right\|_{H_{00}^{\frac{1}{2}}\left(\Gamma_{(u)}\right)^{\prime}} \\
& +\sqrt{\frac{3}{\alpha_{1}}}\left(\sum_{j=1}^{n} \tau_{j}\left(\left\|\boldsymbol{f}^{j}\right\|_{L^{2}(\Omega)^{d}}^{2}+c_{0}^{2}\left\|p_{b}^{j}\right\|_{H^{\frac{1}{2}\left(\Gamma_{(p)}\right)}}^{2}+\alpha_{2}^{2}\left\|g^{j}\right\|_{H_{00}^{\frac{1}{2}}\left(\Gamma_{(u)}\right)^{\prime}}^{2}\right)\right)^{\frac{1}{2}} .
\end{aligned}
$$

and

$$
\begin{aligned}
& \left(\sum_{j=1}^{n} \tau_{j}\left\|\frac{\boldsymbol{u}^{j}-\boldsymbol{u}^{j-1}}{\tau_{j}}\right\|_{L^{2}(\Omega)^{d}}^{2}\right)^{\frac{1}{2}} \\
& \leq c\left(\left\|\boldsymbol{u}_{0}\right\|_{L^{2}(\Omega)^{d}}+\left(\sum_{j=1}^{n} \tau_{j}\left\|\boldsymbol{f}^{j}\right\|_{L^{2}(\Omega)^{d}}^{2}\right)^{\frac{1}{2}}+\left(\sum_{j=1}^{n} \tau_{j}\left\|p_{b}^{j}\right\|_{H^{\frac{1}{2}}\left(\Gamma_{(p)}\right)}^{2}\right)^{\frac{1}{2}}\right. \\
& \left.\quad+\left(\sum_{j=0}^{n} \tau_{j}\left\|g^{j}\right\|_{H_{00}^{\frac{1}{2}\left(\Gamma_{(u)}\right)^{\prime}}}^{2}\right)^{\frac{1}{2}}+\left(\sum_{j=1}^{n} \tau_{j}\left\|\frac{g^{j}-g^{j-1}}{\tau_{j}}\right\|_{H_{00}^{\frac{1}{2}\left(\Gamma_{(u)}\right)^{\prime}}}^{2}\right)^{\frac{1}{2}}\right) .
\end{aligned}
$$

Proof. Since the bilinear form $b(\cdot, \cdot)$ satisfies the inf-sup condition (2.9), the semi-discrete problem (3.1) admits a unique solution at each time step. Next, to bound $\boldsymbol{u}^{n}$, we use the same arguments as for the exact problem. It is advisable to lift the boundary datum $p_{b}^{n}$ as in relation (2.11), then

$$
\forall n \geq 1, \quad\left\|p_{b}^{n}\right\|_{H^{1}(\Omega)} \leq c_{0}\left\|p_{b}^{n}\right\|_{H^{\frac{1}{2}}\left(\Gamma_{p}\right)} .
$$

We set $\boldsymbol{u}_{*}^{n}=\boldsymbol{u}^{n}-\boldsymbol{w}^{n}$ and $p_{*}^{n}=p^{n}-p_{b}^{n}$ where $\boldsymbol{w}^{n}=\boldsymbol{w}\left(\cdot, t_{n}\right)$ is defined in (2.12) and satisfies

$$
\left\|\boldsymbol{w}^{n}\right\|_{L^{2}(\Omega)^{d}} \leq\left\|g^{n}\right\|_{H_{00}^{\frac{1}{2}}\left(\Gamma_{u}\right)^{\prime}}
$$

So, if $\left(\boldsymbol{u}^{n}, p^{n}\right)$ is a solution of problem $(3.1), \boldsymbol{u}_{*}^{n}$ belongs to $\mathbf{V}(\Omega)$ and satisfies

$$
\boldsymbol{u}_{*}^{0}=\boldsymbol{u}^{0}-\boldsymbol{w}^{0} \quad \text { in } \Omega,
$$

and at each time step $n \geq 1$,

$$
\begin{aligned}
\forall \boldsymbol{v} \in \mathbf{V}(\Omega), \quad\left(\boldsymbol{u}_{*}^{n}, \boldsymbol{v}\right)+\tau_{n}\left(\alpha_{*}\left(p_{*}^{n-1}\right) \boldsymbol{u}_{*}^{n}, \boldsymbol{v}\right) & =\tau_{n}\left(\boldsymbol{f}^{n}, \boldsymbol{v}\right)+\left(\boldsymbol{u}_{*}^{n-1}, \boldsymbol{v}\right) \\
& -\tau_{n}\left(\alpha_{*}\left(p_{*}^{n-1}\right) \boldsymbol{w}^{n}, \boldsymbol{v}\right)-\tau_{n} b\left(\boldsymbol{v}, p_{b}^{n}\right) .
\end{aligned}
$$

We take $\boldsymbol{v}=\boldsymbol{u}_{*}^{n}$ in (3.8) and recall a standard identity and Young's inequality that will be used throughout the paper: For all vectors $\boldsymbol{a}, \boldsymbol{b}$ and nonnegative scalar $\beta>0$

$$
\begin{aligned}
2(\boldsymbol{a}-\boldsymbol{b}, \boldsymbol{a}) & =|\boldsymbol{a}|^{2}-|\boldsymbol{b}|^{2}+|\boldsymbol{a}-\boldsymbol{b}|^{2}, \\
2(\boldsymbol{a}, \boldsymbol{b}) & \leq \frac{1}{\beta} \boldsymbol{a}^{2}+\beta \boldsymbol{b}^{2} .
\end{aligned}
$$

We obtain

$$
\begin{aligned}
\left\|\boldsymbol{u}_{*}^{n}\right\|_{L^{2}(\Omega)^{d}}^{2} & -\left\|\boldsymbol{u}_{*}^{n-1}\right\|_{L^{2}(\Omega)^{d}}^{2}+\left\|\boldsymbol{u}_{*}^{n}-\boldsymbol{u}_{*}^{n-1}\right\|_{L^{2}(\Omega)^{d}}^{2}+2 \alpha_{1} \tau_{n}\left\|\boldsymbol{u}_{*}^{n}\right\|_{L^{2}(\Omega)^{d}}^{2} \\
& \leq 2 \tau_{n}\left(\left\|\boldsymbol{f}^{n}\right\|_{L^{2}(\Omega)^{d}}+\left|p_{b}^{n}\right|_{H^{1}(\Omega)}+\alpha_{2}\left\|\boldsymbol{w}^{n}\right\|_{L^{2}(\Omega)^{d}}\right)\left\|\boldsymbol{u}_{*}^{n}\right\|_{L^{2}(\Omega)^{d}} .
\end{aligned}
$$

We obtain the inequality (3.3) by combining these inequalities with inequalities (3.5) and (3.6) and by summing on the $n$.

To derive (3.4), we choose $\boldsymbol{v}=\boldsymbol{u}_{*}^{n}-\boldsymbol{u}_{*}^{n-1}$ in (3.8), this gives

$$
\begin{aligned}
& \left\|\boldsymbol{u}_{*}^{n}-\boldsymbol{u}_{*}^{n-1}\right\|_{L^{2}(\Omega)^{d}}^{2} \\
& \quad \leq \tau_{n}\left(\left\|\boldsymbol{f}^{n}\right\|_{L^{2}(\Omega)^{d}}+\alpha_{2}\left\|\boldsymbol{u}_{*}^{n}\right\|_{L^{2}(\Omega)^{d}}+\alpha_{2}\left\|\boldsymbol{w}^{n}\right\|_{L^{2}(\Omega)^{d}}+\left|p_{b}^{n}\right|_{H^{1}(\Omega)}\right)\left\|\boldsymbol{u}_{*}^{n}-\boldsymbol{u}_{*}^{n-1}\right\|_{L^{2}(\Omega)^{d}}
\end{aligned}
$$


Then, (3.11) becomes

$$
\begin{aligned}
\frac{1}{2} \| \boldsymbol{u}_{*}^{n} & -\boldsymbol{u}_{*}^{n-1} \|_{L^{2}(\Omega)^{d}}^{2} \\
& \leq \frac{4}{2} \tau_{n}^{2}\left(\left\|\boldsymbol{f}^{n}\right\|_{L^{2}(\Omega)^{d}}^{2}+\left|p_{b}^{n}\right|_{H^{1}(\Omega)}^{2}\right)+\frac{4}{2} \alpha_{2}^{2} \tau_{n}^{2}\left(\left\|\boldsymbol{w}^{n}\right\|_{L^{2}(\Omega)^{d}}^{2}+\left\|\boldsymbol{u}_{*}^{n}\right\|_{L^{2}(\Omega)^{d}}^{2}\right) .
\end{aligned}
$$

Then dividing by $\tau_{n}$ and summing over $n$ yield (3.4) for any $n$, with $1 \leq n \leq N$.

Similarly, the sequence of pressures satisfies the following estimate.

Proposition 3.2 For any data $\left(\boldsymbol{f}, g, p_{b}, \boldsymbol{u}_{0}\right)$ satisfying (3.2), the following stability property of the pressure-sequence $\left(p^{n}\right)_{1 \leq n \leq N}$ holds

$$
\begin{aligned}
& \left(\sum_{j=1}^{n} \tau_{j}\left|p^{j}\right|_{H^{1}(\Omega)}^{2}\right)^{\frac{1}{2}} \leq c\left(\left\|\boldsymbol{u}_{0}\right\|_{L^{2}(\Omega)}+\left(\sum_{j=1}^{n} \tau_{j}\left\|\boldsymbol{f}^{j}\right\|_{L^{2}(\Omega)^{d}}^{2}\right)^{\frac{1}{2}}+\left(\sum_{j=1}^{n} \tau_{j}\left\|p_{b}^{j}\right\|_{H^{\frac{1}{2}}\left(\Gamma_{(p)}\right)}^{2}\right)^{\frac{1}{2}}\right. \\
& \left.+\left(\sum_{j=0}^{n} \tau_{j}\left\|g^{j}\right\|_{H_{00}^{\frac{1}{2}}\left(\Gamma_{(u)}\right)^{\prime}}^{2}\right)^{\frac{1}{2}}+\left(\sum_{j=1}^{n} \tau_{j}\left\|\frac{g^{j}-g^{j-1}}{\tau_{j}}\right\|_{H_{00}^{\frac{1}{2}}\left(\Gamma_{(u)}\right)^{\prime}}^{2}\right)^{\frac{1}{2}}\right),
\end{aligned}
$$

where the positive constant $c$ is independent of $n$ and the time step $\tau_{n}$.

Proof. We choose $\boldsymbol{v}=\nabla p^{n}$, in (3.1) and we sum to $n$, we obtain

$$
\sum_{j=1}^{n} \tau_{j}\left|p^{j}\right|_{H^{1}(\Omega)}^{2} \leq 3 \sum_{j=1}^{n}\left(\tau_{j}\left\|\frac{\boldsymbol{u}^{j}-\boldsymbol{u}^{j-1}}{\tau_{j}}\right\|_{L^{2}(\Omega)^{d}}^{2}+\tau_{j}\left\|\boldsymbol{u}^{j}\right\|_{L^{2}(\Omega)^{d}}^{2}+\tau_{j}\left\|\boldsymbol{f}^{j}\right\|_{L^{2}(\Omega)^{d}}^{2}\right) .
$$

Combining this estimate with (3.3) and (3.4) gives the conclusion.

\section{The fully discrete problem}

From now on, we assume that the domain $\Omega$ is the square or the cube $]-1,1\left[{ }^{d}, d=2\right.$ or 3 , and that all data $\boldsymbol{f}, g$ and $p_{b}$ are continuous on $\bar{\Omega} \times[0, T], \bar{\Gamma}_{u} \times[0, T]$ and $\bar{\Gamma}_{p} \times[0, T]$, respectively. We first describe the discrete problem. For each nonnegative integer $m$, we introduce the space $\mathbb{P}_{m}(\Omega)$ of restrictions to $\Omega$ of polynomials with $d$ variables and degree with respect to each variable $\leq m$.

Let $M$ be a fixed positive integer, we introduce the discrete spaces

$$
\mathbb{X}_{M}=\mathbb{P}_{M}(\Omega)^{d}, \quad \mathbb{Y}_{M}=\mathbb{P}_{M}(\Omega), \quad \mathbb{Y}_{M}^{p}=\mathbb{P}_{M}(\Omega) \cap H_{(p)}^{1}(\Omega) .
$$

We recall that there exist a unique set of $M+1$ nodes $\xi_{j}, 0 \leq j \leq M$, with $\xi_{0}=-1$ and $\xi_{M}=1$, and a unique set of $M+1$ weights $\rho_{j}, 0 \leq j \leq M$, such that the following equality holds

$$
\forall \phi \in \mathbb{P}_{2 M-1}(-1,1), \quad \int_{-1}^{1} \phi(\zeta) d \zeta=\sum_{i=0}^{M} \phi\left(\xi_{i}\right) \rho_{i}
$$

We also recall [8, Chap. IV, Cor. 1.10], the following property, which is useful in what follows

$$
\forall \phi_{M} \in \mathbb{P}_{M}(-1,1), \quad\left\|\phi_{M}\right\|_{L^{2}(-1,1)}^{2} \leq \sum_{i=0}^{M} \phi_{M}^{2}\left(\xi_{i}\right) \rho_{i} \leq 3\left\|\phi_{M}\right\|_{L^{2}(-1,1)}^{2} .
$$


We introduce the grid

$$
\Xi= \begin{cases}\left\{\left(\xi_{i}, \xi_{j}\right) ; 0 \leq i, j \leq M\right\} & \text { in the case } d=2 \\ \left\{\left(\xi_{i}, \xi_{j}, \xi_{k}\right) ; \quad 0 \leq i, j, k \leq M\right\} & \text { in the case } d=3\end{cases}
$$

We denote by $\mathcal{I}_{M}$ the Lagrange interpolation operator at the nodes of the grid $\Xi$ with values in $\mathbb{P}_{M}(\Omega)$, and by $\mathcal{I}_{M}^{\Gamma_{u}}$ (resp. $\mathcal{I}_{M}^{\Gamma_{p}}$ ) the Lagrange interpolation operator at the nodes of $\Xi \cap \bar{\Gamma}_{u}$ (resp. $\Xi \cap \bar{\Gamma}_{p}$ ) with values in the space of traces of functions in $\mathbb{P}_{M}(\Omega)$ on $\Gamma_{u}$ (resp. $\left.\Gamma_{p}\right)$.

Finally, we introduce the discrete product, for all continuous functions $u$ and $v$ on $\bar{\Omega}$ by

$$
(u, v)_{M}= \begin{cases}\sum_{i=0}^{M} \sum_{j=0}^{M} u\left(\xi_{i}, \xi_{j}\right) v\left(\xi_{i}, \xi_{j}\right) \rho_{i} \rho_{j} & \text { if } \quad d=2 \\ \sum_{i=0}^{M} \sum_{j=0}^{M} \sum_{k=0}^{M} u\left(\xi_{i}, \xi_{j}, \xi_{k}\right) v\left(\xi_{i}, \xi_{j}, \xi_{k}\right) \rho_{i} \rho_{j} \rho_{k} & \text { if } \quad d=3 .\end{cases}
$$

It follows from (4.3) that it is a scalar product on $\mathbb{P}_{M}(\Omega)$. We denote by $\|\cdot\|_{M}$ the discrete norm which is associated with this scalar product. On each edge or face $\Gamma_{\ell}$ of $\Omega$, we define a discrete product: For instance, if $\Gamma_{\ell}$ is the edge $\left.\{-1\} \times\right]-1,1\left[{ }^{d-1}\right.$

$$
(u, v)_{M}^{(\ell)}= \begin{cases}\sum_{j=0}^{M} u\left(\xi_{0}, \xi_{j}\right) v\left(\xi_{0}, \xi_{j}\right) \rho_{j} & \text { if } \quad d=2, \\ \sum_{j=0}^{M} \sum_{k=0}^{M} u\left(\xi_{0}, \xi_{j}, \xi_{k}\right) v\left(\xi_{0}, \xi_{j}, \xi_{k}\right) \rho_{j} \rho_{k} & \text { if } \quad d=3 .\end{cases}
$$

A global product on $\Gamma_{u}$ is then defined by

$$
(u, v)_{M}^{\Gamma_{u}}=\sum_{\ell \in \mathcal{L}}(u, v)_{M}^{(\ell)}
$$

where $\mathcal{L}$ stands for the set of indices $\ell$ such that $\Gamma_{\ell}$ is contained in $\Gamma_{u}$.

We denote by $p_{M}^{0}$ the image of $p^{0}$ by the operator $\mathcal{I}_{M}$ where $p^{0}$ is defined in Section 3 . We are now in a position to state the discrete problem associated with problem (1.1) and constructed from problem (2.4) to 2.7 by the Galerkin method with numerical integration. It reads as follows:

$$
\begin{aligned}
& \begin{array}{l}
\text { Find }\left(\boldsymbol{u}_{M}^{n}, p_{M}^{n}\right) \in \mathbb{X}_{M} \times \mathbb{Y}_{M} \text { solution of } \\
\boldsymbol{u}_{M}^{0}=\mathcal{I}_{M} \boldsymbol{u}_{0} \quad \text { in } \Omega
\end{array} \\
& \text { and for } 1 \leq n \leq N, \quad p_{M}^{n}=\mathcal{I}_{M}^{\Gamma_{p} p_{b}^{n} \quad \text { on } \Gamma_{p},} \\
& \forall \boldsymbol{v}_{M} \in \mathbb{X}_{M}, \quad\left(\frac{\boldsymbol{u}_{M}^{n}-\boldsymbol{u}_{M}^{n-1}}{\tau_{n}}, \boldsymbol{v}_{M}\right)_{M}+\left(\alpha\left(p_{M}^{n-1}\right) \boldsymbol{u}_{M}^{n}, \boldsymbol{v}_{M}\right)_{M} \\
& \forall q_{M} \in \mathbb{Y}_{M}^{p}, \quad b_{M}\left(\boldsymbol{u}_{M}^{n}, q_{M}\right)=\left(g^{n}, q_{M}\right)_{M}^{\Gamma_{u}}, \quad+b_{M}\left(\boldsymbol{v}_{M}, p_{M}^{n}\right)=\left(\boldsymbol{f}^{n}, \boldsymbol{v}_{M}\right)_{M},
\end{aligned}
$$

where the bilinear form $b_{M}(\cdot, \cdot)$ is defined by $b_{M}(\boldsymbol{v}, q)=(\boldsymbol{v}, \nabla q)_{M}$.

So, this form is continuous in $\mathbb{X}_{M} \times \mathbb{Y}_{M}$ and satisfies the inf-sup condition (that is easily derived by choosing $\boldsymbol{v}_{M}=\nabla q_{M}$ and using (4.3))

$$
\forall q_{M} \in \mathbb{Y}_{M}, \quad \sup _{\boldsymbol{v}_{M} \in \mathbb{X}_{M}} \frac{b_{M}\left(\boldsymbol{v}_{M}, q_{M}\right)}{\left\|\boldsymbol{v}_{M}\right\|_{L^{2}(\Omega)^{d}}} \geq\left|q_{M}\right|_{H^{1}(\Omega)} .
$$


As in the continuous case, its kernel defined by:

$$
\mathbf{V}_{M}(\Omega)=\left\{\boldsymbol{v}_{M} \in \mathbb{X}_{M} ; \quad \forall q_{M} \in \mathbb{Y}_{M}^{p}, \quad b_{M}\left(\boldsymbol{v}_{M}, q_{M}\right)=0\right\} .
$$

By the same arguments as in Section 3 combined with (4.3), we deduce the following theorem.

Theorem 4.1 For any data $\left(\boldsymbol{f}, g, p_{b}, \boldsymbol{u}_{0}\right)$ continuous where needed and satisfying (3.2), problem (4.7) has a unique solution $\left(\boldsymbol{u}_{M}^{n}, p_{M}^{n}\right)$ in $\mathbb{X}_{M} \times \mathbb{Y}_{M}$. Furthermore, this solution satisfies the following stability property

$$
\begin{array}{r}
\left\|\boldsymbol{u}_{M}^{n}\right\|_{L^{2}(\Omega)^{d}}+\left(\sum_{j=1}^{n} \tau_{j}\left\|p_{M}^{j}\right\|_{H^{1}(\Omega)}^{2}\right)^{\frac{1}{2}} \leq c\left(\left\|\mathcal{I}_{M} \boldsymbol{u}_{0}\right\|_{L^{2}(\Omega) d}+\left(\sum_{j=1}^{n} \tau_{j}\left\|\mathcal{I}_{M} \boldsymbol{f}^{j}\right\|_{L^{2}(\Omega)^{d}}^{2}\right)^{\frac{1}{2}}\right. \\
\left.+\left(\sum_{j=1}^{n} \tau_{j}\left\|\mathcal{I}_{M}^{\Gamma_{p}} p_{b}^{j}\right\|_{H^{\frac{1}{2}}\left(\Gamma_{p}\right)}^{2}\right)^{\frac{1}{2}}+\left(\sum_{j=1}^{n} \tau_{j}\left\|\mathcal{I}_{M}^{\Gamma_{u}} g^{j}\right\|_{L^{2}\left(\Gamma_{u}\right)}^{2}\right)^{\frac{1}{2}}\right)
\end{array}
$$

\section{A priori error estimate in the linear case}

To apply the theorem due to Brezzi, Rappaz and Raviart [11], we need to write another formulation of the continuous problem. We define a linear operator $\mathcal{T}$, which is associated with any data $\left(\boldsymbol{f}, g, p_{b}, \boldsymbol{u}_{0}\right)$ satisfying $(2.3)$, the solution $U=(\boldsymbol{u}, p)$ of the following problem

$$
\left\{\begin{aligned}
\forall \boldsymbol{v} \in L^{2}(\Omega)^{d}, & \left(\partial_{t} \boldsymbol{u}, \boldsymbol{v}\right)+\alpha_{0}(\boldsymbol{u}, \boldsymbol{v})+b(\boldsymbol{v}, p)=(\boldsymbol{f}, \boldsymbol{v}), \\
\forall q \in H_{(p)}^{1}(\Omega), & b(\boldsymbol{u}, q)=<g, q>_{\Gamma_{u}}, \\
\left.\boldsymbol{u}\right|_{t=0}=\boldsymbol{u}_{0} \quad \text { in } \Omega & \text { and } \left.p=p_{b} \quad \text { on } \Gamma_{p} \times\right] 0, T[
\end{aligned}\right.
$$

where $\alpha_{0}$ is a nonnegative constant. It is readily checked that problem (5.1) has a unique solution, see [6, Thm. 2.1].

Indeed, we associate a mapping $G$ from $H^{1}\left(0, T ; L^{2}(\Omega)^{d}\right) \times L^{2}\left(0, T ; H^{1}(\Omega)\right)$ into

$$
L^{2}\left(0, T ; L^{2}(\Omega)^{d}\right) \times L^{2}\left(0, T ; H_{00}^{\frac{1}{2}}\left(\Gamma_{u}\right)^{\prime}\right) \times L^{2}\left(0, T ; H^{\frac{1}{2}}\left(\Gamma_{p}\right)\right) \times L^{2}(\Omega)^{d},
$$

defined by

$$
G(U)=\left(\boldsymbol{f}-\left(\alpha(p)-\alpha_{0}\right) \boldsymbol{u}, g, p_{b}, \boldsymbol{u}_{0}\right)
$$

and we set

$$
\mathcal{F}(U)=U-\mathcal{T} G(U)
$$

With this notation, it is obviously checked that the problem (2.4) to (2.7) can equivalently be written as

$$
\mathcal{F}(U)=0
$$

\subsection{About the time discretization}

For $1 \leq n \leq N$, we denote by $\boldsymbol{u}_{\tau}$ and $p_{\tau}$ the functions which are affine on each interval $\left[t_{n-1}, t_{n}\right]$ and equal to $\boldsymbol{u}^{n}$ and $p^{n}$ respectively, at each time $t_{n}$. Also, with any $\boldsymbol{v}$ continuous on $[0, T]$, we introduce the function $\pi_{\tau} \boldsymbol{v}$ which is constant and equal to $\boldsymbol{v}\left(t_{n}\right)$ on each interval $\left.] t_{n-1}, t_{n}\right]$. The semi-discrete operator $\mathcal{T}_{\tau}$ is defined as follow: For any data $\left(\boldsymbol{f}, g, p_{b}, \boldsymbol{u}_{0}\right)$ satisfying (3.2), we denote by $\mathcal{T}_{\tau}\left(\boldsymbol{f}, g, p_{b}, \boldsymbol{u}_{0}\right)$ the pair $\left(\boldsymbol{u}_{\tau}, p_{\tau}\right)$ which is associated with the solution of the semi-discrete problem $\left(\boldsymbol{u}^{n}, p^{n}\right)$ in $L^{2}(\Omega)^{d} \times H^{1}(\Omega)$

$$
\begin{cases}\forall \boldsymbol{v} \in L^{2}(\Omega)^{d}, & \left(\boldsymbol{u}^{n}, \boldsymbol{v}\right)+\tau_{n} \alpha_{0}\left(\boldsymbol{u}^{n}, \boldsymbol{v}\right)+\tau_{n} b\left(\boldsymbol{v}, p^{n}\right)=\tau_{n}\left(\boldsymbol{f}^{n}, \boldsymbol{v}\right)+\left(\boldsymbol{u}^{n-1}, \boldsymbol{v}\right), \\ \forall q \in H_{(p)}^{1}(\Omega), & b\left(\boldsymbol{u}^{n}, q\right)=<g^{n}, q>_{\Gamma_{u}} \\ \boldsymbol{u}^{0}=\boldsymbol{u}_{0} \text { in } \Omega & \text { and } \quad p^{n}=p_{b}^{n} \text { on } \Gamma_{p}\end{cases}
$$


We define the space $\mathbb{W}=\mathcal{C}^{0}\left(0, T ; L^{2}(\Omega)^{d}\right) \times L^{2}\left(0, T ; H^{1}(\Omega)\right)$, equipped with its "standard" norm $\|\cdot\|_{\mathbb{W}}=\left(\|\cdot\|_{\mathcal{C}^{0}\left(0, T ; L^{2}(\Omega)^{d}\right)}^{2}+\|\cdot\|_{L^{2}\left(0, T ; H^{1}(\Omega)\right)}^{2}\right)^{\frac{1}{2}}$. We can now state the following result.

Proposition 5.1 The operator $\mathcal{T}_{\tau}$ satisfies the stability property

$$
\left\|\mathcal{T}_{\tau}(\boldsymbol{f}, 0,0,0)\right\|_{\mathbb{W}} \leq c\left\|\pi_{\tau} \boldsymbol{f}\right\|_{L^{2}\left(0, T ; L^{2}(\Omega)^{d}\right)} .
$$

Proof. In the case of zero boundary conditions and zero initial condition, it is easy to check (5.6) by taking successively $\boldsymbol{v}$ equal to $\boldsymbol{u}^{n}$ and equal to $\nabla p^{n}$ in the first equation of (5.5).

We now intend to estimate the error between the semi-discrete scheme (5.5) and the continuous problem (5.1). Thus, at time $t_{n}$, we write the error equation obtained by subtracting (5.5) from (5.1). We introduce the sequence defined by $\boldsymbol{e}^{n}=\boldsymbol{u}\left(\cdot, t_{n}\right)-\boldsymbol{u}^{n}$.

Obviously, this error satisfies: $\boldsymbol{e}^{0}=\mathbf{0}$ and for $1 \leq n \leq N$,

$$
\begin{aligned}
\forall \boldsymbol{v} \in L^{2}(\Omega)^{d}, \quad\left(\boldsymbol{e}^{n}, \boldsymbol{v}\right)+\alpha_{0} \tau_{n}\left(\boldsymbol{e}^{n}, \boldsymbol{v}\right) & +\tau_{n} b\left(\boldsymbol{v}, p\left(\cdot, t_{n}\right)-p^{n}\right) \\
& =\tau_{n}\left(\boldsymbol{\varepsilon}^{n}, \boldsymbol{v}\right)+\left(\boldsymbol{e}^{n-1}, \boldsymbol{v}\right) \\
\forall q \in H_{(p)}^{1}(\Omega), \quad b\left(\boldsymbol{e}^{n}, q\right) & =0,
\end{aligned}
$$

where the consistency error $\varepsilon^{n}$ is given by

$$
\varepsilon^{n}=\frac{\boldsymbol{u}\left(\cdot, t_{n}\right)-\boldsymbol{u}\left(\cdot, t_{n-1}\right)}{\tau_{n}}-\partial_{t} \boldsymbol{u}\left(\cdot, t_{n}\right) .
$$

We refer to [6, Prop. 3.2 \& Cor. 3.3] for the proof of the next proposition.

Proposition 5.2 Assume that problem (5.1) admits the solution (u,p) such that the velocity $\boldsymbol{u}$ belongs to $H^{2}\left(0, T ; L^{2}(\Omega)^{d}\right)$, then the following a priori error estimates hold for $1 \leq n \leq N$

$$
\begin{aligned}
\left\|\boldsymbol{e}^{n}\right\|_{L^{2}(\Omega)^{d}} & \leq \frac{1}{\sqrt{3 \alpha_{0}}}|\tau|\|\boldsymbol{u}\|_{H^{2}\left(0, t_{n} ; L^{2}(\Omega)^{d}\right)}, \\
\left(\sum_{j=1}^{n} \tau_{j}\left\|\frac{\boldsymbol{e}^{j}-\boldsymbol{e}^{j-1}}{\tau_{j}}\right\|_{L^{2}(\Omega)^{d}}^{2}\right)^{\frac{1}{2}} & \leq \frac{1}{2 \sqrt{3}}|\tau|\|\boldsymbol{u}\|_{H^{2}\left(0, t_{n} ; L^{2}(\Omega)^{d}\right)}, \\
\left(\sum_{j=1}^{n} \tau_{j}\left|p\left(\cdot, t_{j}\right)-p^{j}\right|_{H^{1}(\Omega)}^{2}\right)^{\frac{1}{2}} & \leq \frac{1}{\sqrt{3}}|\tau|\|\boldsymbol{u}\|_{H^{2}\left(0, t_{n} ; L^{2}(\Omega)^{d}\right)} .
\end{aligned}
$$

Note that the equivalence between the discrete and continuous norms of the error is due to the regularity of $u$. Thus, a direct consequence of (5.6) and (5.9) is

Theorem 5.3 For any $\boldsymbol{f} \in \mathcal{C}^{0}\left(0, T ; L^{2}(\Omega)^{d}\right)$,

$$
\lim _{|\tau| \rightarrow 0}\left\|\left(\mathcal{T}-\mathcal{T}_{\tau}\right)(\boldsymbol{f}, 0,0,0)\right\|_{\mathbb{W}}=0
$$

\subsection{About the space discretization}

Similarly, we denote by $\boldsymbol{u}_{M \tau}$ (reps. $p_{M \tau}$ ) the function which is affine on each interval $\left[t_{n-1}, t_{n}\right]$ and equal to $\boldsymbol{u}_{M}^{n}$ (resp. $p_{M}^{n}$ ) at each time $t_{n}, 1 \leq n \leq N$. We now define the discrete operator $\mathcal{T}_{M \tau}$ as follows:

For any data $\boldsymbol{f}, g$ and $p_{b}$ continuous respectively on $\bar{\Omega} \times[0, T], \bar{\Gamma}_{u} \times[0, T]$ and $\bar{\Gamma}_{p} \times[0, T]$ and $\boldsymbol{u}_{0}$ in $L^{2}(\Omega)^{d}$, we set

$$
\mathcal{T}_{M \tau}\left(\boldsymbol{f}, g, p_{b}, \boldsymbol{u}_{0}\right)=\tilde{U}_{M \tau}=\left(\boldsymbol{u}_{M \tau}, p_{M \tau}\right)
$$


where $\left(\boldsymbol{u}_{M \tau}, p_{M \tau}\right)$ is associated with the solution of the following problem $\left(\boldsymbol{u}_{M}^{n}, p_{M}^{n}\right)$

$$
\left\{\begin{array}{rr}
\boldsymbol{u}_{M}^{0}=\mathcal{I}_{M} \boldsymbol{u}_{0} & \text { in } \Omega \quad \text { and } \quad p_{M}^{n}=\mathcal{I}_{M}^{\Gamma_{p}} p_{b}^{n} \quad \text { on } \Gamma_{p} \\
\forall \boldsymbol{v}_{M} \in \mathbb{X}_{M}, & \left(\boldsymbol{u}_{M}^{n}, \boldsymbol{v}_{M}\right)_{M}+\tau_{n} \alpha_{0}\left(\boldsymbol{u}_{M}^{n}, \boldsymbol{v}_{M}\right)_{M}+\tau_{n} b_{M}\left(\boldsymbol{v}_{M}, p_{M}^{n}\right) \\
= & \tau_{n}\left(\boldsymbol{f}^{n}, \boldsymbol{v}_{M}\right)+\left(\boldsymbol{u}_{M}^{n-1}, \boldsymbol{v}_{M}\right)_{M} \\
\forall q_{M} \in \mathbb{Y}_{M}^{p}, & b_{M}\left(\boldsymbol{u}_{M}^{n}, q_{M}\right)=<g^{n}, q_{M}>_{\Gamma_{u}}
\end{array}\right.
$$

The stability of the operator $\mathcal{T}_{M \tau}$ is easily derived by using the same arguments in Proposition 5.1.

Proposition 5.4 For any continuous datum $\boldsymbol{f}$ in $\bar{\Omega} \times[0, T]$, there exists a nonnegative constant $c$, such that the following stability property of $\mathcal{T}_{M \tau}$ holds

$$
\left\|\mathcal{T}_{M \tau}(\boldsymbol{f}, 0,0,0)\right\|_{\mathbb{W}} \leq c \sum_{j=1}^{n} \tau_{j} \sup _{\boldsymbol{v}_{M} \in \mathbb{X}_{M}} \frac{\int_{\Omega} \boldsymbol{f}\left(\boldsymbol{x}, t_{j}\right) \boldsymbol{v}_{M}\left(\boldsymbol{x}, t_{j}\right) d \boldsymbol{x}}{\left\|\boldsymbol{v}_{M}\right\|_{L^{2}(\Omega)^{d}}} .
$$

Theorem 5.5 Assume that $\boldsymbol{f}, g$ and $p_{b}$ are continuous on $\bar{\Omega} \times[0, T], \bar{\Gamma}_{u} \times[0, T]$ and $\bar{\Gamma}_{p} \times[0, T]$ respectively, and also that the velocity $\boldsymbol{u}$ solution of problem $(5.1)$ belongs to $H^{2}\left(0, T ; H^{s}(\Omega)^{d}\right)$ and that the boundary value $p_{b}$ belongs to $\mathcal{C}^{0}\left(0, T ; H^{\sigma+\frac{1}{2}}\left(\Gamma_{p}\right)\right)$ for a real numbers $s \geq 1$ and $\sigma>\frac{1}{2}$. Then, the following error estimate between the velocity solution $\boldsymbol{u}_{\tau}$ of problem (5.5) and the velocity solution $\boldsymbol{u}_{M \tau}$ of problem (5.11) holds

$$
\begin{aligned}
& \left\|\boldsymbol{u}_{\tau}-\boldsymbol{u}_{M \tau}\right\|_{\mathcal{C}^{0}\left(0, T ; L^{2}(\Omega)^{d}\right)} \\
& \quad \leq c_{1}\left(|\tau|+M^{-s}\right)\|\boldsymbol{u}\|_{H^{2}\left(0, T ; H^{s}(\Omega)^{d}\right)}+c_{2} M^{-\sigma}\left\|p_{b}\right\|_{\mathcal{C}^{0}\left(0, T ; H^{\left.\sigma+\frac{1}{2}\left(\Gamma_{p}\right)\right)}\right.} .
\end{aligned}
$$

Proof. This rather technical proof requires some preliminary results. We introduce the subspace $\mathbb{X}_{M}^{g}$ of functions in $\mathbb{X}_{M}$ such that their traces on $\Gamma_{u}$ are equal to $\mathcal{I}_{M}^{\Gamma_{u}} g$. Let $\boldsymbol{v}_{M}^{n}$ belongs to $\mathbb{X}_{M}^{g}$, then $\boldsymbol{u}_{M}^{n}-\boldsymbol{v}_{M}^{n}$ belongs to the discrete kernel space $\mathbf{V}_{M}(\Omega)$. The goal of the first step is to bound the spectral error $\left\|\boldsymbol{u}^{n}-\boldsymbol{u}_{M}^{n}\right\|_{M}$ at each time step. First, by choosing $\boldsymbol{u}_{M}^{n}-\boldsymbol{v}_{M}^{n}$ as a test function in (5.11) and using (3.9), it is readily checked that the first equation in (5.11) can be rewritten as follow:

$$
\begin{gathered}
\frac{1}{2 \tau_{n}}\left(\left\|\boldsymbol{u}_{M}^{n}-\boldsymbol{v}_{M}^{n}\right\|_{M}^{2}-\left\|\boldsymbol{u}_{M}^{n-1}-\boldsymbol{v}_{M}^{n-1}\right\|_{M}^{2}+\left\|\left(\boldsymbol{u}_{M}^{n}-\boldsymbol{v}_{M}^{n}\right)-\left(\boldsymbol{u}_{M}^{n-1}-\boldsymbol{v}_{M}^{n-1}\right)\right\|_{M}^{2}\right) \\
+\alpha_{0}\left\|\boldsymbol{u}_{M}^{n}-\boldsymbol{v}_{M}^{n}\right\|_{M}^{2}+\alpha_{0}\left(\boldsymbol{v}_{M}^{n}, \boldsymbol{u}_{M}^{n}-\boldsymbol{v}_{M}^{n}\right)_{M}+b_{M}\left(\boldsymbol{u}_{M}^{n}-\boldsymbol{v}_{M}^{n}, p_{M}^{n}\right) \\
=\left(\boldsymbol{f}^{n}, \boldsymbol{u}_{M}^{n}-\boldsymbol{v}_{M}^{n}\right)-\left(\frac{\boldsymbol{v}_{M}^{n}-\boldsymbol{v}_{M}^{n-1}}{\tau_{n}}, \boldsymbol{u}_{M}^{n}-\boldsymbol{v}_{M}^{n}\right)_{M} .
\end{gathered}
$$

We next use the semi-discrete formulation (5.5) in order to replace $\left(\boldsymbol{f}^{n}, \boldsymbol{u}_{M}^{n}-\boldsymbol{v}_{M}^{n}\right)$ by its equivalent, this yields

$$
\begin{gathered}
\frac{1}{2 \tau_{n}}\left(\left\|\boldsymbol{u}_{M}^{n}-\boldsymbol{v}_{M}^{n}\right\|_{M}^{2}-\left\|\boldsymbol{u}_{M}^{n-1}-\boldsymbol{v}_{M}^{n-1}\right\|_{M}^{2}+\left\|\left(\boldsymbol{u}_{M}^{n}-\boldsymbol{v}_{M}^{n}\right)-\left(\boldsymbol{u}_{M}^{n-1}-\boldsymbol{v}_{M}^{n-1}\right)\right\|_{M}^{2}\right) \\
+\alpha_{0}\left\|\boldsymbol{u}_{M}^{n}-\boldsymbol{v}_{M}^{n}\right\|_{M}^{2}+\alpha_{0}\left(\boldsymbol{v}_{M}^{n}, \boldsymbol{u}_{M}^{n}-\boldsymbol{v}_{M}^{n}\right)_{M}+b_{M}\left(\boldsymbol{u}_{M}^{n}-\boldsymbol{v}_{M}^{n}, p_{M}^{n}\right) \\
=\left(\frac{\boldsymbol{u}^{n}-\boldsymbol{u}^{n-1}}{\tau_{n}}, \boldsymbol{u}_{M}^{n}-\boldsymbol{v}_{M}^{n}\right)+\alpha_{0}\left(\boldsymbol{u}^{n}, \boldsymbol{u}_{M}^{n}-\boldsymbol{v}_{M}^{n}\right)+b\left(\boldsymbol{u}_{M}^{n}-\boldsymbol{v}_{M}^{n}, p^{n}\right) \\
-\left(\frac{\boldsymbol{v}_{M}^{n}-\boldsymbol{v}_{M}^{n-1}}{\tau_{n}}, \boldsymbol{u}_{M}^{n}-\boldsymbol{v}_{M}^{n}\right)_{M} .
\end{gathered}
$$


As in Section 3 and owing to Bernardi et al [8, Thm. III.3.1], there exists a lifting $p_{b M}$ in $\mathbb{Y}_{M}$ of the boundary datum $p_{b}$ on $\Gamma_{p}$, such that

$$
p_{b M}=\mathcal{I}_{M}^{\Gamma_{p}} p_{b} \quad \text { on } \quad \Gamma_{p},
$$

and it satisfies

$$
\left\|p_{b M}^{n}\right\|_{H^{1}(\Omega)} \leq c\left\|\mathcal{I}_{M}^{\Gamma_{p}} p_{b}^{n}\right\|_{H^{\frac{1}{2}\left(\Gamma_{p}\right)}}, \quad \forall n \geq 1,
$$

where $c$ denote some positive constant.

Subtracting the quantity $b_{M}\left(\boldsymbol{u}_{M}^{n}-\boldsymbol{v}_{M}^{n}, p_{b M}^{n}\right)$ in both side of equation (5.14), and observing that $b_{M}\left(\boldsymbol{u}_{M}^{n}-\boldsymbol{v}_{M}^{n}, p_{M}^{n}-p_{b M}^{n}\right)$ is equal to 0 , we obtain

$$
\begin{aligned}
\frac{1}{2 \tau_{n}}\left(\left\|\boldsymbol{u}_{M}^{n}-\boldsymbol{v}_{M}^{n}\right\|_{M}^{2}-\left\|\boldsymbol{u}_{M}^{n-1}-\boldsymbol{v}_{M}^{n-1}\right\|_{M}^{2}\right. & \left.+\left\|\left(\boldsymbol{u}_{M}^{n}-\boldsymbol{v}_{M}^{n}\right)-\left(\boldsymbol{u}_{M}^{n-1}-\boldsymbol{v}_{M}^{n-1}\right)\right\|_{M}^{2}\right) \\
& +\alpha_{0}\left\|\boldsymbol{u}_{M}^{n}-\boldsymbol{v}_{M}^{n}\right\|_{M}^{2}=\mathbf{I}_{\mathbf{1}}+\mathbf{I}_{\mathbf{2}}+\mathbf{I}_{\mathbf{3}}+\mathbf{I}_{\mathbf{4}}
\end{aligned}
$$

with

$$
\begin{aligned}
& \mathbf{I}_{\mathbf{1}}=\left(\frac{\left(\boldsymbol{u}^{n}-\boldsymbol{v}_{M}^{n}\right)-\left(\boldsymbol{u}^{n-1}-\boldsymbol{v}_{M}^{n-1}\right)}{\tau_{n}}, \boldsymbol{u}_{M}^{n}-\boldsymbol{v}_{M}^{n}\right)+\alpha_{0}\left(\boldsymbol{u}^{n}-\boldsymbol{v}_{M}^{n}, \boldsymbol{u}_{M}^{n}-\boldsymbol{v}_{M}^{n}\right), \\
& \mathbf{I}_{\mathbf{2}}=\left(\frac{\boldsymbol{v}_{M}^{n}-\boldsymbol{v}_{M}^{n-1}}{\tau_{n}}, \boldsymbol{u}_{M}^{n}-\boldsymbol{v}_{M}^{n}\right)-\left(\frac{\boldsymbol{v}_{M}^{n}-\boldsymbol{v}_{M}^{n-1}}{\tau_{n}}, \boldsymbol{u}_{M}^{n}-\boldsymbol{v}_{M}^{n}\right)_{M}, \\
& \mathbf{I}_{\mathbf{3}}=\alpha_{0}\left(\left(\boldsymbol{v}_{M}^{n}, \boldsymbol{u}_{M}^{n}-\boldsymbol{v}_{M}^{n}\right)-\left(\boldsymbol{v}_{M}^{n}, \boldsymbol{u}_{M}^{n}-\boldsymbol{v}_{M}^{n}\right)_{M}\right), \\
& \mathbf{I}_{\mathbf{4}}=b\left(\boldsymbol{u}_{M}^{n}-\boldsymbol{v}_{M}^{n}, p^{n}\right)-b_{M}\left(\boldsymbol{u}_{M}^{n}-\boldsymbol{v}_{M}^{n}, p_{b M}^{n}\right) .
\end{aligned}
$$

Bounding the quantity $\mathbf{I}_{\mathbf{1}}$ results from the estimate of the approximation error. To evaluate all quantities $\mathbf{I}_{\mathbf{j}}, j=2, \ldots, 4$, we use the standard arguments for the error issued from numerical integration (see $[8$, Chap. VI] for instance). All this gives the desired result.

Remark 5.6 By the same arguments as previously we can also prove the estimate which we need later on

$$
\begin{gathered}
\alpha_{0} \sum_{j=1}^{n} \tau_{j}\left\|\boldsymbol{u}^{j}-\boldsymbol{v}_{M}^{j}\right\|_{M}^{2} \leq c M^{-s}\left(\left(\sum_{j=1}^{n} \tau_{j}\left\|\boldsymbol{u}^{j}\right\|_{H^{s}(\Omega)^{d}}^{2}\right)^{\frac{1}{2}}+\left(\sum_{j=1}^{n} \tau_{j}\left\|\frac{\boldsymbol{u}^{j}-\boldsymbol{u}^{j-1}}{\tau_{j}}\right\|_{H^{s}(\Omega)^{d}}^{2}\right)^{\frac{1}{2}}\right) \\
+c M^{-\sigma}\left(\sum_{j=1}^{n} \tau_{j}\left\|p_{b}^{j}\right\|_{H^{\sigma+\frac{1}{2}\left(\Gamma_{p}\right)}}^{2}\right)^{\frac{1}{2}} .
\end{gathered}
$$

Theorem 5.7 Under the same assumptions as for Theorem 5.5, we assume that the pressure solution $p$ of problem (5.1) belongs to $L^{2}\left(0, T ; H^{s+1}(\Omega)\right)$. The following pressure error estimate holds

$$
\begin{gathered}
\left\|p_{\tau}-p_{M \tau}\right\|_{L^{2}\left(0, T ; H^{1}(\Omega)\right)} \leq c\left(M^{-s}+|\tau|\right)\left(\|u\|_{H^{1}\left(0, T ; H^{s}(\Omega) d\right.}+\|p\|_{L^{2}\left(0, T ; H^{s+1}(\Omega)\right)}\right) \\
+c M^{-\sigma}\left\|p_{b}\right\|_{\mathcal{C}^{0}\left(0, T ; H^{\sigma+\frac{1}{2}}\left(\Gamma_{p}\right)\right)} .
\end{gathered}
$$

Proof. 1) For any function $q_{M}^{n} \in \mathbb{Y}_{M}$, we take $\boldsymbol{v}_{M}=\nabla\left(p_{M}^{n}-q_{M}^{n}\right)$ as a test function in (5.11) and we replace the term $\left(\boldsymbol{f}^{n}, \nabla\left(p_{M}^{n}-q_{M}^{n}\right)\right)$ by its equivalent in the semi-discrete formulation (5.5). Then, we can write

$$
\begin{aligned}
\left\|\nabla\left(p_{M}^{n}-q_{M}^{n}\right)\right\|_{M}^{2} & =\left(\frac{\boldsymbol{u}^{n}-\boldsymbol{u}^{n-1}}{\tau_{n}}, \nabla\left(p_{M}^{n}-q_{M}^{n}\right)\right)-\left(\frac{\boldsymbol{u}_{M}^{n}-\boldsymbol{u}_{M}^{n-1}}{\tau_{n}}, \nabla\left(p_{M}^{n}-q_{M}^{n}\right)\right)_{M} \\
& +b\left(\nabla\left(p_{M}^{n}-q_{M}^{n}\right), p^{n}-q_{M}^{n}\right)+b\left(\nabla\left(p_{M}^{n}-q_{M}^{n}\right), q_{M}^{n}\right)+b_{M}\left(\nabla\left(p_{M}^{n}-q_{M}^{n}\right), q_{M}^{n}\right) \\
& +\alpha_{0}\left(\left(\boldsymbol{u}^{n}, \nabla\left(p_{M}^{n}-q_{M}^{n}\right)\right)-\left(\boldsymbol{u}^{n}, \nabla\left(p_{M}^{n}-q_{M}^{n}\right)\right)_{M}\right)
\end{aligned}
$$


We obtain by same techniques used to bound the velocity error $\left\|\boldsymbol{u}^{n}-\boldsymbol{u}_{M}^{n}\right\|_{L^{2}(\Omega)^{d}}$

$$
\begin{aligned}
& \left\|p^{n}-p_{M}^{n}\right\|_{H^{1}(\Omega)} \leq c M^{-s}\left(\left(\sum_{j=1}^{n} \tau_{j}\left\|\boldsymbol{u}^{j}\right\|_{H^{s}(\Omega)^{d}}^{2}\right)^{\frac{1}{2}}+\left(\sum_{j=1}^{n} \tau_{j}\left\|\frac{\boldsymbol{u}^{j}-\boldsymbol{u}^{j-1}}{\tau_{j}}\right\|_{H^{s}(\Omega)^{d}}^{2}\right)^{\frac{1}{2}}\right. \\
& \left.+\left(\sum_{j=1}^{n} \tau_{j}\left\|\frac{\left(\boldsymbol{u}^{j}-\boldsymbol{u}^{j-1}\right)-\left(\boldsymbol{u}_{M}^{j}-\boldsymbol{u}_{M}^{j-1}\right)}{\tau_{j}}\right\|_{L^{2}(\Omega)^{d}}^{2}\right)^{\frac{1}{2}}\right) \\
& +c\left(M^{-s}\left(\sum_{j=1}^{n} \tau_{j}\left\|p^{j}\right\|_{H^{s}(\Omega)}^{2}\right)^{\frac{1}{2}}+M^{-\sigma}\left(\sum_{j=1}^{n} \tau_{j}\left\|p_{b}^{j}\right\|_{H^{\sigma+\frac{1}{2}\left(\Gamma_{p}\right)}}^{2}\right)^{\frac{1}{2}}\right),
\end{aligned}
$$

2) We want to estimate $\sum_{j=1}^{n} \tau_{j}\left\|\frac{\left(\boldsymbol{u}^{j}-\boldsymbol{u}^{j-1}\right)-\left(\boldsymbol{u}_{M}^{j}-\boldsymbol{u}_{M}^{j-1}\right)}{\tau_{j}}\right\|_{L^{2}(\Omega)^{d}}^{2}$. To this end, we let $\boldsymbol{v}_{M}^{n}$ in $\mathbb{X}_{M}^{g}, \forall n, 0 \leq n \leq N$, and we choose the test function in (5.11) equal to $\hat{\boldsymbol{v}}_{M}=\delta \boldsymbol{u}_{M}-\delta \boldsymbol{v}_{M}$, where $\delta \boldsymbol{v}=\boldsymbol{v}^{n}-\boldsymbol{v}^{n-1}$. We first remark that $\hat{\boldsymbol{v}}_{M}$ belongs to $\mathbf{V}_{M}(\Omega)$. As the previous argument, we write

$$
\begin{aligned}
\frac{1}{\tau_{n}}\left\|\hat{\boldsymbol{v}}_{M}\right\|_{M}^{2} & =\frac{1}{\tau_{n}}\left(\delta \boldsymbol{u}-\delta \boldsymbol{v}_{M}, \hat{\boldsymbol{v}}_{M}\right)+\tau_{n} \alpha_{0}\left(\left(\boldsymbol{u}^{n}, \frac{\hat{\boldsymbol{v}}_{M}}{\tau_{n}}\right)-\left(\boldsymbol{u}_{M}^{n}, \frac{\hat{\boldsymbol{v}}_{M}}{\tau_{n}}\right)_{M}\right) \\
& +\frac{1}{\tau_{n}}\left(\left(\delta \boldsymbol{v}_{M}, \hat{\boldsymbol{v}}_{M}\right)-\left(\delta \boldsymbol{v}_{M}, \hat{\boldsymbol{v}}_{M}\right)_{M}\right) \\
& +\tau_{n}\left(b\left(\frac{\hat{\boldsymbol{v}}_{M}}{\tau_{n}}, p_{b}^{n}\right)-b_{M}\left(\frac{\hat{\boldsymbol{v}}_{M}}{\tau_{n}}, p_{b M}^{n}\right)\right) .
\end{aligned}
$$

We introduce the quantities $\mathcal{I}_{M-1} \boldsymbol{u}^{n}$ and $\mathcal{I}_{M-1} \delta \boldsymbol{u}$ with obvious notation, and we apply the Cauchy-Schwarz inequality. We obtain, for all $q_{M-1}$ in $\mathbb{Y}_{M-1}$

$$
\begin{aligned}
\frac{1}{\tau_{n}}\left\|\hat{\boldsymbol{v}}_{M}\right\|_{M}^{2} \leq c( & \frac{1}{\tau_{n}}\left\|\delta \boldsymbol{u}-\delta \boldsymbol{v}_{M}\right\|_{L^{2}(\Omega)^{d}}^{2}+\tau_{n}\left(\left\|\boldsymbol{u}^{n}-\mathcal{I}_{M-1} \boldsymbol{u}^{n}\right\|_{L^{2}(\Omega)^{d}}^{2}\right. \\
+ & \tau_{n}\left\|\boldsymbol{u}^{n}-\boldsymbol{u}_{M}^{n}\right\|_{L^{2}(\Omega)^{d}}^{2}+\frac{1}{\tau_{n}}\left\|\delta \boldsymbol{u}-\mathcal{I}_{M-1} \delta \boldsymbol{u}\right\|_{L^{2}(\Omega)^{d}}^{2} \\
& \left.+\tau_{n}\left\|p_{b}^{n}-p_{b M}^{n}\right\|_{H^{1}(\Omega)^{d}}^{2}+\tau_{n}\left\|p_{b}^{n}-q_{M-1}\right\|_{H^{1}(\Omega)}\right) .
\end{aligned}
$$

3) To finish this poof, we choose $q_{M-1}$ to be the image of $p_{b}^{n}$ under the orthogonal projection operator from $H^{1}(\Omega)$ to $\mathbb{Y}_{M-1}$. This gives

$$
\begin{aligned}
\sum_{j=1}^{n} \tau_{j}\left\|p^{j}-p_{M}^{j}\right\|_{H^{1}(\Omega)}^{2} \leq & c\left(M ^ { - 2 s } \left(\sum_{j=0}^{n} \tau_{j}\left\|\boldsymbol{u}^{j}\right\|_{H^{s}(\Omega)^{d}}^{2}+\sum_{j=1}^{n} \tau_{j}\left\|\frac{\boldsymbol{u}^{j}-\boldsymbol{u}^{j-1}}{\tau_{j}}\right\|_{H^{s}(\Omega)^{d}}^{2}\right.\right. \\
& \left.\left.+\sum_{j=1}^{n} \tau_{j}\left\|p^{j}\right\|_{H^{s+1}(\Omega)}^{2}\right)+M^{-2 \sigma} \sum_{j=1}^{n} \tau_{j}\left\|p_{b}^{j}\right\|_{H^{\sigma+\frac{1}{2}\left(\Gamma_{p}\right)}}^{2}\right) .
\end{aligned}
$$

which is the desired result.

We deduce the convergence property owing to Theorems 5.5 and 5.7.

Theorem 5.8 For any datum $\boldsymbol{f}$ in $\mathcal{C}^{0}\left(0, T ; L^{2}(\Omega)^{d}\right)$,

$$
\lim _{|\tau| \rightarrow 0} \lim _{M \rightarrow \infty}\left\|\left(\mathcal{T}_{\tau}-\mathcal{T}_{M \tau}\right)(\boldsymbol{f}, 0,0,0)\right\|_{\mathbb{W}}=0
$$




\section{A priori error estimate for the full problem}

The aim of this section is to prove optimal error estimates. To this aim, we check the assumptions of the theorem due to Brezzi, Rappaz and Raviart [11].

We observe that problem (4.7) can equivalently be written

$$
\mathcal{F}_{M \tau}\left(U_{M \tau}\right)=U_{M \tau}-\mathcal{T}_{M \tau} G_{M \tau}\left(U_{M \tau}\right)=0
$$

where the mapping $G_{M \tau}$ is defined by the components $G_{M \tau}^{i}, i=1,2,3,4$, which are given by

$$
\begin{aligned}
& <G_{M \tau}^{1}\left(U_{M \tau}\right), V_{M}>=\left(\boldsymbol{f}-\left(\alpha\left(p_{M}^{n-1}\right)-\alpha_{0}\right) \boldsymbol{u}_{M \tau}, \boldsymbol{v}_{M}\right)_{M}, \\
& <G_{M \tau}^{2}\left(U_{M \tau}\right), V_{M}>=\left(g, q_{M}\right)_{M}^{\Gamma_{u}}, \\
& G_{M \tau}^{3}\left(U_{M \tau}\right)=p_{b} \quad \text { and } \quad G_{M \tau}^{4}\left(U_{M \tau}\right)=\boldsymbol{u}_{0} .
\end{aligned}
$$

Let us choose an approximation $V_{M \tau}=\left(\boldsymbol{v}_{M \tau}, q_{M \tau}\right)$ of the solution $U=(\boldsymbol{u}, p)$ in $\mathbb{X}_{M} \times \mathbb{Y}_{M}$ which satisfies the following approximation properties, for appropriate real numbers $\ell, s$ such that $0 \leq \ell \leq s$,

$$
\begin{aligned}
\left\|\boldsymbol{u}_{\tau}-\boldsymbol{v}_{M \tau}\right\|_{\mathcal{C}^{0}\left(0, T ; H^{\ell}(\Omega)^{d}\right)} & \leq c M^{\ell-s}\|\boldsymbol{u}\|_{\mathcal{C}^{0}\left(0, T ; H^{s}(\Omega)^{d}\right)} \\
\left\|p_{\tau}-q_{M \tau}\right\|_{L^{2}\left(0, T ; H^{\ell+1}(\Omega)\right)} & \leq c M^{\ell-s}\|p\|_{L^{2}\left(0, T ; H^{s+1}(\Omega)\right)}
\end{aligned}
$$

The existence of such an approximation is stated in [7, Theorem 7.4] (see also [8, Chap. III, Thm. 2.4 \& Chap. VI, Thm. 2.5]). From now on, we denote by $D$ the differential operator with respect to $U$.

Assumption 6.1 The solution $U=(\boldsymbol{u}, p)$ of problem (2.4) to (2.7)

(i) belongs to $H^{2}\left(0, T ; H^{s}(\Omega)^{d}\right) \times L^{2}\left(0, T ; H^{s+1}(\Omega)\right)$, for some $s>0$ in dimension $d=2$ and some $s>\frac{1}{2}$ in dimension $d=3$,

(ii) is such that $D \mathcal{F}(U)$ is an isomorphism of the space $\mathbb{W}$.

In a first step, we must prove the analogue of part (ii) of Assumption 6.1 for the discrete operator. Let us denote by $\mathcal{E}(\mathbb{W})$ the space of endomorphisms of $\mathbb{W}$. We introduce the fully discrete space $\mathbb{W}_{M \tau}$ of a pair $(\boldsymbol{v}, q)$ of functions which are affine on each interval $\left[t_{n-1}, t_{n}\right]$, $1 \leq n \leq N$ and such that their values in $t_{j}$ belong to $\mathbb{X}_{M} \times \mathbb{Y}_{M}$. It is readily checked that this space is finite dimensional and imbedded in $\mathbb{W}$.

Lemma 6.2 Assume that the mapping $\alpha(\cdot)$ is of class $\mathcal{C}^{2}$ with bounded derivatives. If Assumption 6.1 holds, there exist positive real numbers $\tau_{0}$ and $M_{0}$ such that for all $\tau,|\tau| \leq \tau_{0}$, and for all $M \geq M_{0}$, the operator $D \mathcal{F}_{M \tau}\left(V_{M \tau}\right)$ is an isomorphism of $\mathbb{W}_{M \tau}$ and the norm of its inverse is bounded independently of $\tau$ and $M$.

Proof. We observe that

$$
\begin{aligned}
D \mathcal{F}_{M \tau}\left(V_{M \tau}\right)= & D \mathcal{F}(U)+\left(\mathcal{T}-\mathcal{T}_{M \tau}\right) D G(U) \\
& +\mathcal{T}_{M \tau}\left(D G(U)-D G\left(V_{M \tau}\right)\right)+\mathcal{T}_{M \tau}\left(D G\left(V_{M \tau}\right)-D G_{M \tau}\left(V_{M \tau}\right)\right) .
\end{aligned}
$$

So we now prove that the last three terms in the right hand side tend to zero when $|\tau| \rightarrow 0$ and $M \rightarrow \infty$.

1) Let us first observe that, for any $W_{M \tau}=\left(\boldsymbol{w}_{M \tau}, r_{M \tau}\right)$ in $\mathbb{W}_{M \tau}$,

$$
D G(U) \cdot W_{M \tau}=\left(-\left(\alpha(p)-\alpha_{0}\right) \boldsymbol{w}_{M \tau}-\alpha^{\prime}(p) r_{M \tau} \boldsymbol{u}, 0,0,0\right) .
$$


Owing to Theorem 1.1' in [10], the mapping $p \mapsto \alpha(p)$ is continuous from $H^{s+1}(\Omega)$ into itself, where $s$ is smaller enough, then in the subset to $\mathcal{C}^{0}\left(0, T ; L^{2}(\Omega)^{d}\right)$. Since $\boldsymbol{w}_{M \tau}$ is bounded in $L^{2}(\Omega)^{d}$, the quantity $\left(\alpha(p)-\alpha_{0}\right) \boldsymbol{w}_{M \tau}$ belongs to $\mathcal{C}^{0}\left(0, T ; L^{2}(\Omega)^{d}\right)$. Thanks to Assumption 6.1, $\boldsymbol{u}$ belongs to $L^{\rho}(\Omega)^{d}$, with $\rho>2$ in dimension $d=2$ and $\rho>3$ in dimension $d=3$. Setting $\frac{1}{\rho}=\frac{1-s}{2}$ and $\frac{1}{\rho}+\frac{1}{\rho^{\prime}}=\frac{1}{2}$, we use the compact imbedding of $H^{1}(\Omega)$ in $L^{\rho^{\prime}}(\Omega)$, to derive that the quantity $\alpha^{\prime}(p) r_{M \tau} \boldsymbol{u}$ belongs to a compact set of $L^{2}(\Omega)^{d}$. Thanks to the expansion: $\mathcal{T}-\mathcal{T}_{M \tau}=\left(\mathcal{T}-\mathcal{T}_{\tau}\right)+\left(\mathcal{T}_{\tau}-\mathcal{T}_{M \tau}\right),(5.10)$ and $(5.21)$, we derive

$$
\left\|\left(\mathcal{T}-\mathcal{T}_{M \tau}\right)(D G(U))\right\|_{\mathcal{E}(\mathbb{W})} \rightarrow 0, \quad \text { when }|\tau| \rightarrow 0 \quad \text { and } \quad M \rightarrow \infty
$$

2) Combining the Lipschitz property of $\alpha(\cdot)$ and $\alpha^{\prime}(\cdot)$ with the imbedding of $H^{1}(\Omega)$ in $L^{\rho^{\prime}}(\Omega)$, we obtain

$$
\begin{aligned}
& \left\langle\left(D G(U)-D G\left(V_{M \tau}\right)\right) \cdot W_{M \tau}, Z\right\rangle \\
& \quad \leq c\left(\left\|p-q_{M \tau}\right\|_{H^{1}(\Omega)}\left\|\boldsymbol{w}_{M \tau}\right\|_{L^{\rho}(\Omega)^{d}}\right. \\
& \left.\quad+\left\|p-q_{M \tau}\right\|_{H^{1}(\Omega)}\left\|r_{M \tau} \boldsymbol{u}\right\|_{L^{\rho}(\Omega)^{d}}+\left\|r_{M \tau}\right\|_{L^{\infty}(\Omega)}\left\|\boldsymbol{u}-\boldsymbol{v}_{M \tau}\right\|_{L^{2}(\Omega)^{d}}\right)\|\boldsymbol{z}\|_{L^{2}(\Omega)^{d}} .
\end{aligned}
$$

Owing to standard inverse inequalities [15], $\left(\boldsymbol{w}_{M \tau}, r_{M \tau}\right)$ is bounded in $L^{2}(\Omega)^{d} \times H^{1}(\Omega)$ and according to (5.12) and (6.2), we deduce that

$$
\lim _{|\tau| \rightarrow 0 M \rightarrow \infty} \lim _{M \tau}\left\|\mathcal{T}_{M \tau}\left(D G(U)-D G\left(V_{M \tau}\right)\right)\right\|_{\mathcal{E}(\mathbb{W})}=0 .
$$

3) Finally, to bound the last term, we observe that

$$
\begin{aligned}
& \left\langle\left(D G(U)-D G_{M \tau}\left(V_{M \tau}\right)\right) \cdot W_{M \tau}, Z_{M}\right\rangle \\
& =\alpha_{0}\left(\left(\boldsymbol{w}_{M \tau}, \boldsymbol{z}_{M}\right)-\left(\boldsymbol{w}_{M \tau}, \boldsymbol{z}_{M}\right)_{M}\right) \\
& +\quad\left(\left(-\alpha\left(q_{M \tau}\right) \boldsymbol{w}_{M \tau}, \boldsymbol{z}_{M}\right)-\left(-\alpha\left(q_{M \tau}\right) \boldsymbol{w}_{M \tau}, \boldsymbol{z}_{M}\right)_{M}\right) \\
& \quad-\left(\left(\alpha^{\prime}\left(q_{M \tau}\right) r_{M \tau} \boldsymbol{v}_{M \tau}, \boldsymbol{z}_{M}\right)-\left(\alpha^{\prime}\left(q_{M \tau}\right) r_{M \tau} \boldsymbol{v}_{M \tau}, \boldsymbol{z}_{M}\right)_{M}\right) .
\end{aligned}
$$

Since the arguments for evaluating the three terms are similar, we only consider the third one which is the more complex. We set $M^{\diamond}$ equal to the integer part of $\frac{M-1}{3}$ and we introduce the approximations $\beta_{M^{\diamond} \tau}$ of $\alpha^{\prime}\left(q_{M \tau}\right)$ and $r_{M^{\diamond} \tau}$ of $r_{M \tau}$ in $\mathbb{Y}_{M^{\diamond}}$ and $\boldsymbol{v}_{M^{\diamond} \tau}$ of $\boldsymbol{u}$ in $\mathbb{X}_{M^{\diamond}}$, then

$$
\begin{aligned}
\left(\alpha^{\prime}\left(q_{M \tau}\right) r_{M \tau} \boldsymbol{v}_{M \tau}, \boldsymbol{z}_{M}\right)- & \left(\alpha^{\prime}\left(q_{M \tau}\right) r_{M \tau} \boldsymbol{v}_{M \tau}, \boldsymbol{z}_{M}\right)_{M} \\
\leq & c\left(\left\|\alpha^{\prime}\left(q_{M \tau}\right) r_{M \tau} \boldsymbol{v}_{M \tau}-\beta_{M^{\diamond} \tau} r_{M^{\diamond} \tau} \boldsymbol{v}_{M^{\diamond} \tau}\right\|_{L^{2}(\Omega)^{d}}\right. \\
& \left.\quad+\left\|\mathcal{I}_{M}\left(\alpha^{\prime}\left(q_{M \tau}\right)\right) r_{M \tau} \boldsymbol{v}_{M \tau}-\beta_{M^{\diamond} \tau} r_{M^{\diamond} \tau} \boldsymbol{v}_{M^{\triangleright} \tau}\right\|_{L^{2}(\Omega)^{d}}\right)\left\|\boldsymbol{z}_{M}\right\|_{L^{2}(\Omega)^{d}}
\end{aligned}
$$

Thus, by using the imbedding of $H^{1}(\Omega)$ into any $L^{q}(\Omega), q<\infty$ in dimension $d=2$, into $L^{6}(\Omega)$ in dimension $d=3$ and some stability properties of the polynomials $\beta_{M^{\diamond} \tau}, r_{M^{\diamond} \tau}$ and $v_{M^{\diamond} \tau}$ which can be found in $[8$, Chap. III], the desired result is now easy.

We refer to [3, Lem. 3.5] for a detailed proof of the next lemma.

Lemma 6.3 If Assumption 6.1 holds and the mapping $\alpha(\cdot)$ is of class $\mathcal{C}^{2}$ with bounded derivatives, there exist a neighbourhood of $U_{M \tau}$ in $\mathbb{W}_{M \tau}$ and a positive constant $c$, such that the following property holds for any $Z_{M}$ in this neighbourhood

$$
\left\|D F_{M \tau}\left(V_{M \tau}\right)-D F_{M \tau}\left(Z_{M}\right)\right\|_{\mathcal{E}\left(\mathbb{W}_{M \tau}\right)} \leq c\left\|V_{M \tau}-Z_{M}\right\|_{\mathbb{W}}
$$

We now evaluate the quantity $\left\|\mathcal{F}_{M \tau}\left(V_{M \tau}\right)\right\|_{\mathbb{W}}$. 
Lemma 6.4 If the assumptions of the previous lemma hold, and if the data $\left(\boldsymbol{f}, p_{b}, g\right)$ belongs to $\mathcal{C}^{0}\left(0, T ; H^{\sigma}(\Omega)^{d}\right) \times \mathcal{C}^{0}\left(0, T ; H^{\sigma+\frac{1}{2}}\left(\Gamma_{p}\right)\right) \times \mathcal{C}^{0}\left(0, T ; H^{\sigma}\left(\Gamma_{u}\right)\right), \sigma>\frac{d}{2}$, then the following estimate is satisfied

$$
\begin{aligned}
\left\|\mathcal{F}_{M \tau}\left(V_{M \tau}\right)\right\|_{\mathbb{W}} \leq & c(\boldsymbol{u})\left(M^{-s}+|\tau|\right)\left(\|\boldsymbol{u}\|_{H^{2}\left(0, T ; H^{s}(\Omega)^{d}\right)}+\|p\|_{L^{2}\left(0, T ; H^{s+1}(\Omega)\right)}\right) \\
& +c M^{-\sigma}\left(\|\boldsymbol{f}\|_{\mathcal{C}^{0}\left(0, T ; H^{\sigma}(\Omega)^{d}\right)}+\left\|p_{b}\right\|_{\mathcal{C}^{0}\left(0, T ; H^{\left.\sigma+\frac{1}{2}\left(\Gamma_{p}\right)\right)}\right.}+\|g\|_{\mathcal{C}^{0}\left(0, T ; H^{\sigma}\left(\Gamma_{u}\right)\right.}\right) .
\end{aligned}
$$

Proof. Recalling that $\mathcal{F}(U)=0$, we have

$$
\begin{aligned}
& \left\|\mathcal{F}_{M \tau}\left(V_{M \tau}\right)\right\|_{\mathbb{W}} \leq\left\|U-V_{M \tau}\right\|_{\mathbb{W}}+\left\|\left(\mathcal{T}-\mathcal{T}_{M \tau}\right) G(U)\right\|_{\mathbb{W}} \\
& \quad+\left\|\mathcal{T}_{M \tau}\left(G(U)-G\left(V_{M \tau}\right)\right)\right\|_{\mathbb{W}}+\| \mathcal{T}_{M \tau}\left(G\left(V_{M \tau}\right)-G_{M \tau}\left(V_{M \tau}\right) \|_{\mathbb{W}}\right.
\end{aligned}
$$

The first and the second terms are evaluated in (6.2) and also in (5.9), (5.13) and (5.20). In order to evaluate the third one, we consider $Z_{M}$ in the unit sphere of $\mathbb{W}_{M \tau}$, and we write

$$
\begin{aligned}
<G(U)-G\left(V_{M \tau}\right)(\cdot, & \left.t_{n}\right), Z_{M}\left(\cdot, t_{n}\right)> \\
= & \left(\left(\alpha(p)-\alpha\left(q_{M \tau}\right)\right)\left(\cdot, t_{n}\right) \boldsymbol{u}\left(\cdot, t_{n}\right), \boldsymbol{z}_{M}\left(\cdot, t_{n}\right)\right) \\
& +\left(\alpha\left(q_{M \tau}\right)\left(\cdot, t_{n}\right)\left(\boldsymbol{u}-\boldsymbol{v}_{M \tau}\right)\left(\cdot, t_{n}\right), \boldsymbol{z}_{M}\left(\cdot, t_{n}\right)\right) \\
& +\alpha_{0}\left(\left(\boldsymbol{u}-\boldsymbol{v}_{M \tau}\right)\left(\cdot, t_{n}\right), \boldsymbol{z}_{M}\left(\cdot, t_{n}\right)\right) .
\end{aligned}
$$

The same arguments as in the previous proofs yield that

$$
\begin{aligned}
<G(U)-G\left(V_{M \tau}\right)\left(\cdot, t_{n}\right), & Z_{M}\left(\cdot, t_{n}\right)> \\
\leq c & \left\|\left(p-q_{M \tau}\right)\left(\cdot, t_{n}\right)\right\|_{H^{1}(\Omega)}\left\|\boldsymbol{u}\left(\cdot, t_{n}\right)\right\|_{L^{\rho}(\Omega)^{d}} \\
& \left.\quad+\left\|\alpha\left(q_{M \tau}\right)\left(\cdot, t_{n}\right)\right\|_{L^{\infty}(\Omega)}\left\|\left(u-v_{M \tau}\right)\left(\cdot, t_{n}\right)\right\|_{L^{2}(\Omega)^{d}}\right)\left\|z_{M}\left(\cdot, t_{n}\right)\right\|_{L^{2}(\Omega)^{d}} .
\end{aligned}
$$

Thus, owing to (5.12) and (6.2) we deduce

$$
\left\|\mathcal{T}_{M \tau}\left(G(U)-G\left(V_{M \tau}\right)\right)\right\|_{\mathbb{W}} \leq c(\boldsymbol{u}) M^{-s} \sum_{n=1}^{N} \tau_{n}\left(\left\|\boldsymbol{u}\left(\cdot, t_{n}\right)\right\|_{H^{s}(\Omega)^{d}}+\left\|p\left(\cdot, t_{n}\right)\right\|_{H^{s+1}(\Omega)}\right) .
$$

Finally, for the same reasons as in the proof of Lemma 6.2, the last term is bounded by

$$
\begin{gathered}
\left\|\mathcal{T}_{M \tau}\left(G\left(V_{M \tau}\right)-G_{M \tau}\left(V_{M \tau}\right)\right)\right\|_{\mathbb{W}} \\
\leq c(\boldsymbol{u}) T^{\frac{1}{2}} M^{-s}\left(\left(\sum_{n=1}^{N} \tau_{n}\left\|\boldsymbol{u}\left(\cdot, t_{n}\right)\right\|_{H^{s}(\Omega)^{d}}^{2}\right)^{\frac{1}{2}}+\left(\sum_{n=1}^{N} \tau_{n}\left\|p\left(\cdot, t_{n}\right)\right\|_{H^{s+1}(\Omega)}^{2}\right)^{\frac{1}{2}}\right) \\
+M^{-\sigma}\left(\sum_{n=1}^{N} \tau_{n}\left\|\boldsymbol{f}\left(\cdot, t_{n}\right)\right\|_{H^{\sigma}(\Omega)^{d}}^{2}\right)^{\frac{1}{2}} .
\end{gathered}
$$

This concludes the proof.

Owing to Lemmas 6.2 to 6.4, all the assumptions needed to apply the theorem of Brezzi, Rappaz and Raviart [11] (see also [12, Chap.IV, Thm. 3.1]) are satisfied. This leads to the final a priori error estimate.

Theorem 6.5 If Assumption 6.1 holds and if

$$
\boldsymbol{f} \in \mathcal{C}^{0}\left(0, T ; H^{\sigma}(\Omega)^{d}\right), \quad g \in \mathcal{C}^{0}\left(0, T ; H^{\sigma}\left(\Gamma_{u}\right)\right) \quad \text { and } \quad p_{b} \in \mathcal{C}^{0}\left(0, T ; H^{\sigma+\frac{1}{2}}\left(\Gamma_{p}\right)\right),
$$


$\sigma>\frac{d}{2}$, there exist a neighbourhood of $(\boldsymbol{u}, p)$ in $\mathbb{W}$ and positive real numbers $\tau_{0}$ and $M_{0}$ such that, for all $\tau,|\tau| \leq \tau_{0}$ and for all $M \geq M_{0}$, problem (4.7) has a unique solution $\left(\boldsymbol{u}_{M \tau}, p_{M \tau}\right)$ in this neighbourhood. Moreover this solution satisfies the following a priori error estimate

$$
\begin{aligned}
& \left\|\boldsymbol{u}-\boldsymbol{u}_{M \tau}\right\|_{\mathcal{C}^{0}\left(0, T ; L^{2}(\Omega)^{d}\right)}+\left\|p-p_{M \tau}\right\|_{L^{2}\left(0, T ; H^{1}(\Omega)\right)} \\
& \leq c(\boldsymbol{u})\left(M^{-s}+|\tau|\right)\left(\|\boldsymbol{u}\|_{H^{2}\left(0, T ; H^{s}(\Omega)^{d}\right)}+\|p\|_{L^{2}\left(0, T ; H^{s+1}(\Omega)\right)}\right) \\
& \quad+c M^{-\sigma}\left(\|\boldsymbol{f}\|_{\mathcal{C}^{0}\left(0, T ; H^{\sigma}(\Omega)^{d}\right)}+\left\|p_{b}\right\|_{L^{2}\left(0, T ; H^{\left.\sigma+\frac{1}{2}\left(\Gamma_{p}\right)\right)}\right.}+\|g\|_{\mathcal{C}^{0}\left(0, T ; H^{\sigma}\left(\Gamma_{u}\right)\right.}\right),
\end{aligned}
$$

where the constant $c(\boldsymbol{u})$ depends on $\boldsymbol{u}$.

This estimate is fully optimal. Moreover Assumption 6.1 is not restrictive, it only implies the local uniqueness of the solution $(\boldsymbol{u}, p)$ which seems likely.

\section{Numerical experiments}

In this section numerical results in dimension $d=2$ and $d=3$ are compared to the theoretical convergence results (6.5) provided in previous Sections. To observe these convergence properties we shall use the technique of manufactured solutions. An analytical divergence-free velocity field and a pressure field are substituted in the Darcy's equations to yield balancing volumetric forces terms. The space discretization is performed using a spectral method as previously described (see $[7,8]$ for more details) that we have implemented in FreeFEM3D ${ }^{1}$, see Yakoubi [18] for more details. The results are obtained using a $\mathbb{P}_{M}^{d} \times \mathbb{P}_{M}$ space discretization of $(\boldsymbol{u}, p)$, and the backward Euler discretization with uniform time step $\tau_{n}=\delta t$. The nonlinear term $\alpha(p(\cdot, t))$ is taking equal to $\exp (p(\cdot, t))$.

Finally, at each step, linear systems are obtained and solved using a preconditioned GMRES (Generalized Minimal Residual) iterative routine, see for instance Saad [17].

\subsection{Time accuracy}

The first test is used to validate the time accuracy. We consider Darcy's equation (1.1) in $\Omega=] 0,1\left[^{3}\right.$ and suitable forcing functions such that the exact solution is given by

$$
\left\{\begin{aligned}
u_{1}(x, y, z, t) & =2 y z(y-1)(z-1) \sin (t)+2 x z+y^{3}+z^{4}-1 \\
u_{2}(x, y, z, t) & =-x z(x-1)(z-1) \sin (t)-x(x-1)^{2}+z^{2} \\
u_{3}(x, y, z, t) & =-z^{2} \\
p(x, y, z, t) & =x y z(x-1)(y-1)(z-1) t^{2}
\end{aligned}\right.
$$

In each direction $x, y$ and $z$, the degree $M$ of the polynomials is equal to 6 , so the spatial discretization errors are negligible compared with the time discretization error. We recover a convergence order for velocity and pressure that decreases as the time step tends to 0, which confirms the theoretical estimate obtained by passing in (6.5) to the limit when the polynomial degree $M$ tends to $\infty$ :

$$
\left\|(\boldsymbol{u}, p)-\left(\boldsymbol{u}_{M \tau}, p_{M \tau}\right)\right\|_{\mathcal{C}^{0}\left(0, T ; L^{2}(\Omega)^{d}\right) \times L^{2}\left(0, T ; H^{1}(\Omega)\right)} \leq C \delta t\|(\boldsymbol{u}, p)\|_{H^{2}\left(0, T ; L^{2}(\Omega)^{d}\right) \times L^{2}\left(0, T ; H^{1}(\Omega)\right)} .
$$

At $t=1$, we compute the error

$$
\mathcal{E}_{\delta t}=\left(\left\|\left(\boldsymbol{u}-\boldsymbol{u}_{M}\right)(\cdot, 1)\right\|_{L^{2}(\Omega)^{d}}^{2}+\left\|\left(p-p_{M}\right)(\cdot, 1)\right\|_{H^{1}(\Omega)}^{2}\right)^{1 / 2},
$$

for several time step values $\delta t=0.1, \delta t / 2=0.05$ until $\delta t / 2^{6}=0.0015625$.

By setting $\mathcal{O}_{\frac{\delta t}{2}}=\frac{\log \left(\frac{\mathcal{E}_{\delta t}}{\mathcal{E}_{\frac{\delta}{2}}}\right)}{\log (2)}$, we observe that the convergence rate of order 1 obtained in Table 1 confirms the theoretical results.

\footnotetext{
${ }^{1}$ http://www.freefem.org/ff3d/
} 
Table 1: Estimated convergence order

\begin{tabular}{|c|c|c|c|c|}
\hline$\delta t$ & $\left\|\boldsymbol{u}-\boldsymbol{u}_{M \tau}\right\|_{L^{2}(\Omega)^{3}}$ & $\left\|p-p_{M \tau}\right\|_{H^{1}(\Omega)}$ & $\mathcal{E}_{\delta t}$ & $\mathcal{O}_{\frac{\delta t}{2}}$ \\
\hline $1 / 10$ & 0.00118208 & $3.7977672 \times 10^{-4}$ & $1.2415891 \times 10^{-3}$ & \\
\hline $1 / 20$ & 0.00059374 & $1.9549339 \times 10^{-4}$ & $6.2509682 \times 10^{-4}$ & 0.9900362 \\
\hline $1 / 4$ & 0.00029753 & $0.9914039 \times 10^{-4}$ & $3.1361837 \times 10^{-4}$ & 0.9950695 \\
\hline $1 / 80$ & 0.00014893 & $4.991711 \times 10^{-5}$ & $1.5707563 \times 10^{-4}$ & 0.9975507 \\
\hline $1 / 160$ & $7.45078 \times 10^{-5}$ & $2.504243 \times 10^{-5}$ & $0.7860366 \times 10^{-4}$ & 0.9987909 \\
\hline $1 / 320$ & $3.72639 \times 10^{-5}$ & $1.255598 \times 10^{-5}$ & $0.3932239 \times 10^{-4}$ & 0.9992454 \\
\hline $1 / 640$ & $1.86346 \times 10^{-5}$ & $6.2847384 \times 10^{-6}$ & $0.1966586 \times 10^{-4}$ & 0.9996574 \\
\hline
\end{tabular}

\subsection{Space accuracy}

The aim of the second test is to verify the space accuracy. The computational domain is the square $\Omega=]-1,1\left[^{2}\right.$, and the exact solution of Darcy equation is given as follows:

$$
\left\{\begin{aligned}
u_{1}(x, y, t) & =\sin (t) \sin (\pi x) \cos (\pi y) \\
u_{2}(x, y, t) & =-\sin (t) \cos (\pi x) \sin (\pi y) \\
p(x, y, t) & =t^{2} \sin (\pi x) \cos (\pi y) .
\end{aligned}\right.
$$

We fix the time step $\delta t$ equal to 0.1 , and we plot the $L^{2}$-norm error of the velocity and $H^{1}$-norm error of the pressure between the numerical solution and the exact solution at $t=1$ second with a successive polynomial degree from $M=5$ to $M=25$. It is clear from Figure 1 that the convergence slopes of the velocity and pressure errors coincide with the slope of the function $e^{-x}$. Hence the spectral convergence for all unknowns is obtained which is consistent with the error estimate (6.5) when the time step tends to 0 . We observe that beyond degree 20, the leading error is due to the accuracy of the machine, so that the curve stops decreasing. 


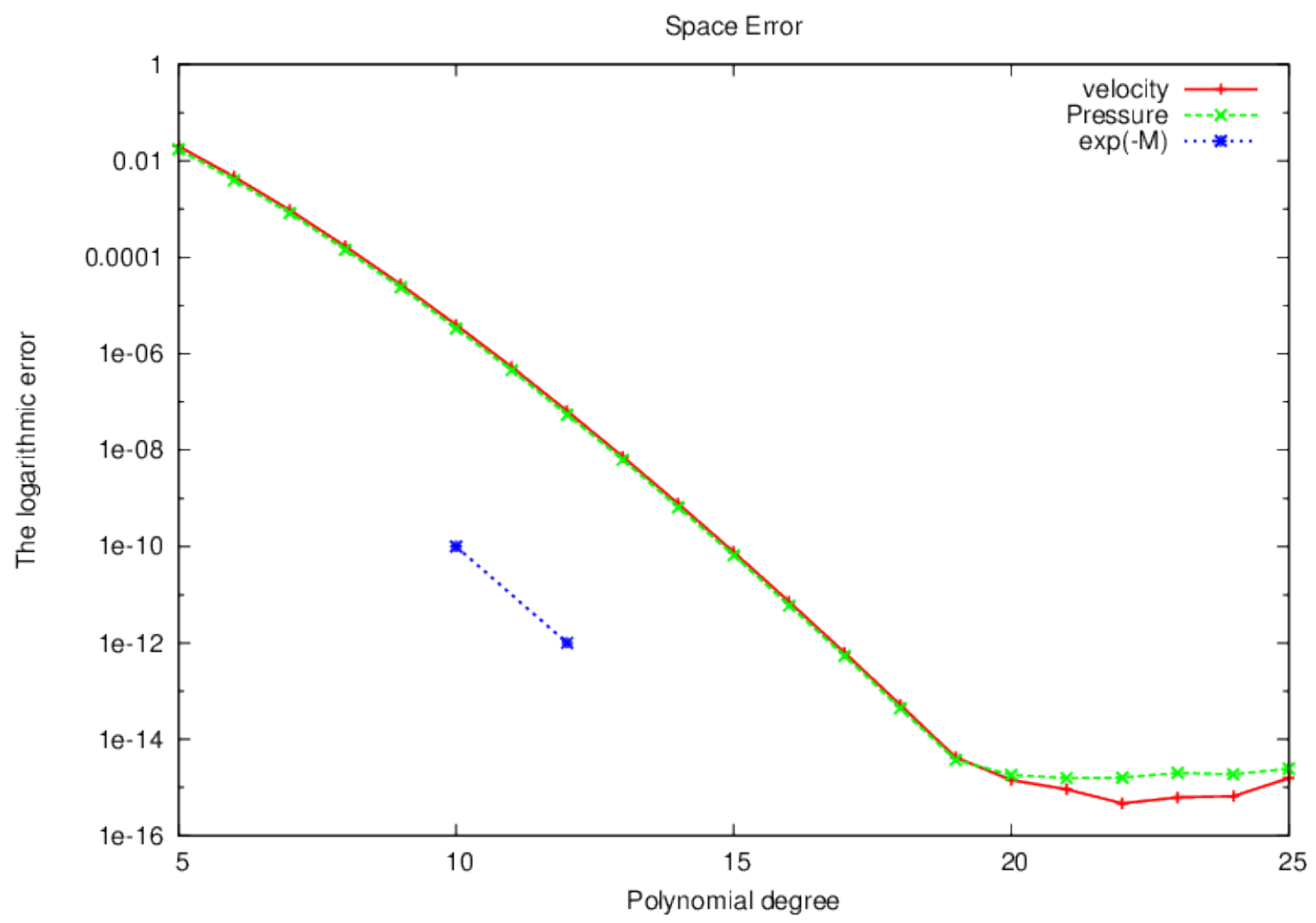

Figure 1: Convergence rate with respect to polynomial degree

In Figures 2 and 3, we present the exact and discrete solutions issued from (7.2), where the discrete one computed with $M=15$.
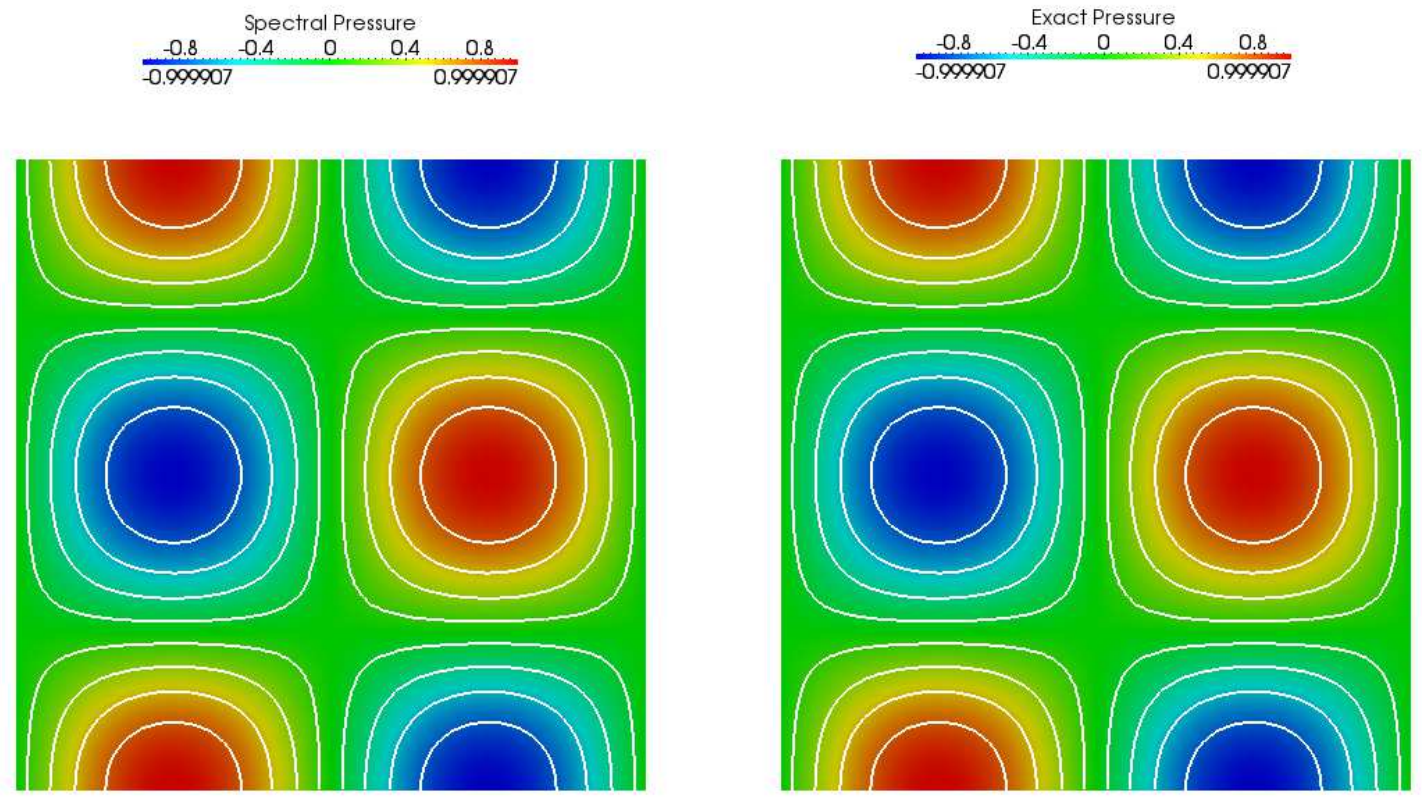

Figure 2: Pressure. Left: Spectral. Right: Exact 

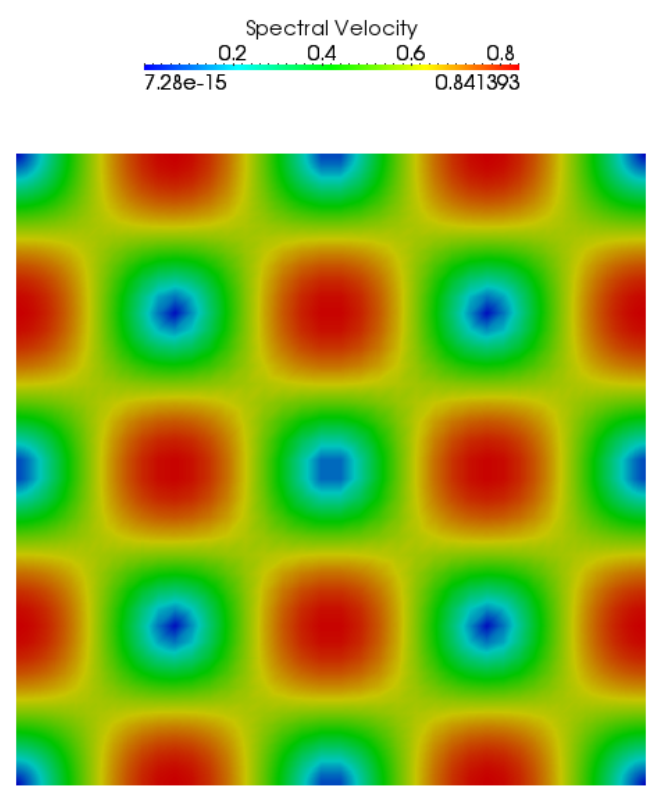

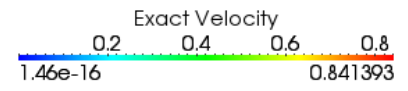

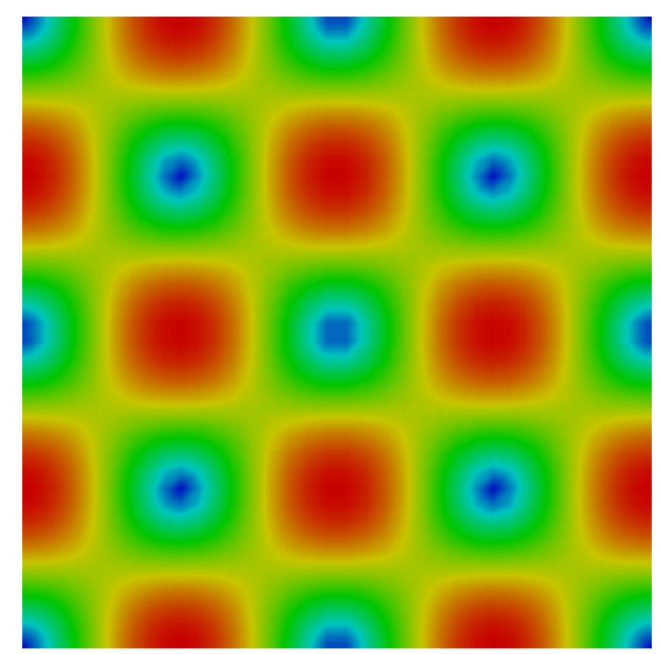

Figure 3: Velocity. Left: Spectral. Right: Exact

Figure 4 presents the fields of the exact and spectral velocity, obtained for $M=15$ in spectral case.

$$
\begin{array}{ccrr}
\multicolumn{4}{c}{\text { Spectral Velocity }} \\
0.2 & 0.4 & 0.6 & 0.8 \\
\hline 7.28 \mathrm{e}-15 & & & 0.841393
\end{array}
$$

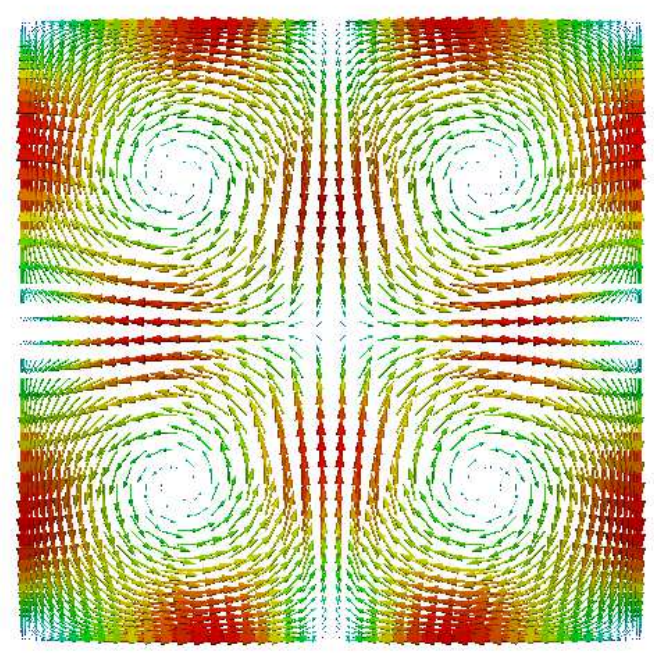

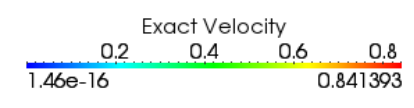

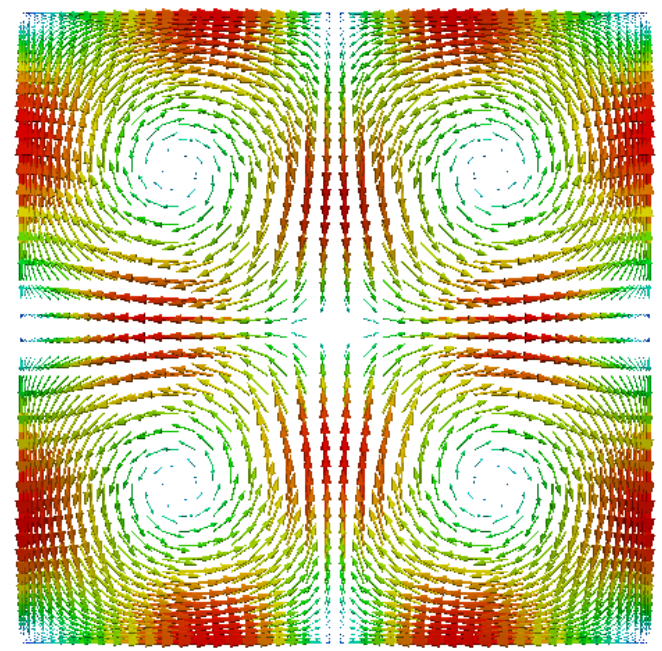

Figure 4: Velocity fields. Left: Spectral. Right: Exact

It can be noted that the left and right parts of the figures can not be distinguished.

\subsection{A more realistic computation}

To conclude, we consider the case where the porous medium occupies the domain

$$
\Omega=]-10,10[\times] 0,1[\times]-1,0[,
$$


located just under the surface. The data are given as follows.

1) The part $\Gamma_{p}$ of the boundary is the top face $]-10,10[\times] 0,1\left[\times\{0\}\right.$. The pressure $p_{b}$ represents the atmospheric pressure and is constant equal to 1 .

2) On $\Gamma_{u}$, the function $g$ has its support contained in the left face $\left.\{-10\} \times\right] 0,1[\times]-1,0[$. More precisely, this support is the disk

$$
x=-10, \quad(y-0.5)^{2}+(z+0.5)^{2} \leq 0.01,
$$

on which it is equal to

$$
g(-10, y, z, t)=\lambda(t)\left(1-100\left((y-0.5)^{2}+(z+0.5)^{2}\right)\right), \quad \text { with } \quad \lambda(t)=\frac{10 t}{t+1} .
$$

3) The datum $\boldsymbol{f}$ and the initial velocity $\boldsymbol{u}_{0}$ are taken equal to zero.

Note that, since the velocity is divergence-free, the integral of its flux on $\partial \Omega$ is zero, so that the fluid goes out through $\Gamma_{p}$.

The computation is performed with time step $\delta t=0.1$ and degrees of polynomials $M$ equal to 20 in the $x$-direction, to 12 in the $y$ - and $z$-directions (this is due to the anisotropy of the domain). Figure 5 presents the isovalues of the pressure at time $t=1$ and from top to bottom in the three planes $z=-0.1, z=-0.5$ and $z=-0.9$. Figure 6 presents the projection of the velocity also at time $t=1$ in the plane $x=-4.5$.
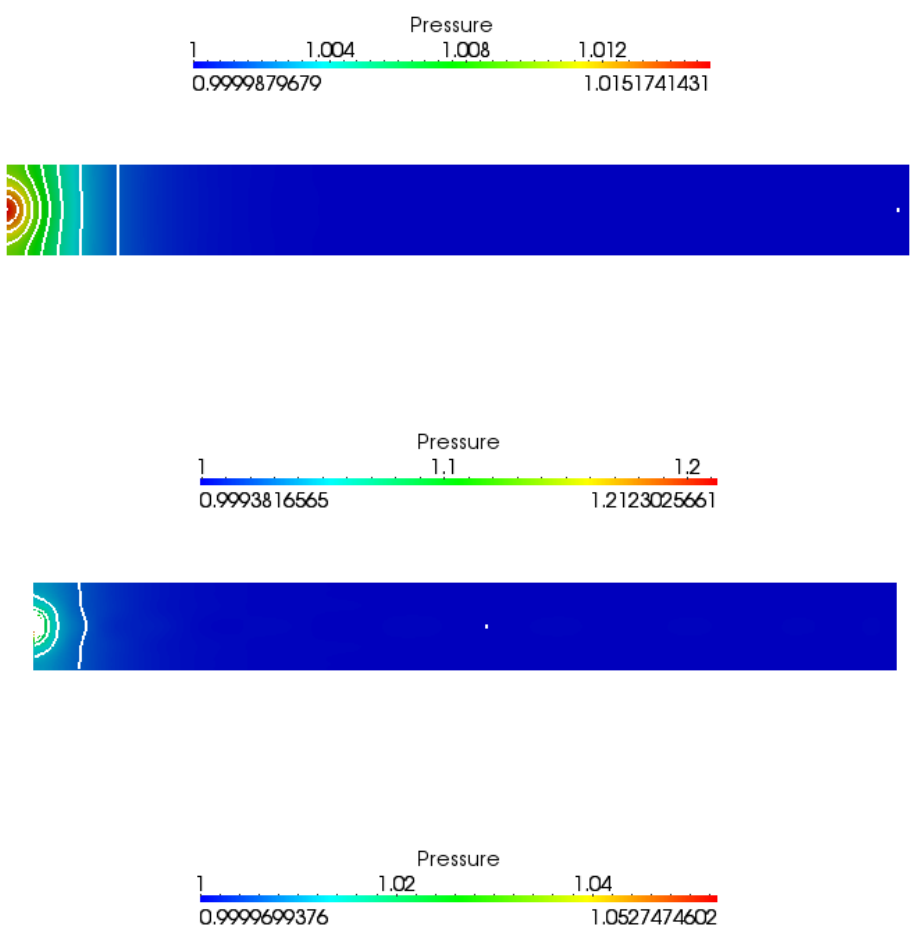

Figure 5: Pressure in three horizontal planes 


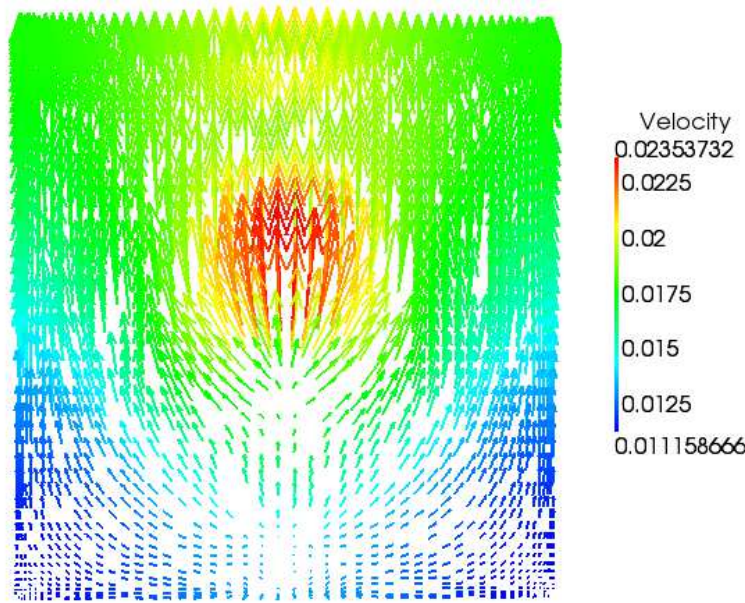

Figure 6: Projection of the velocity in a vertical plane

In this paper, we have analyzed a numerical model for the non linear Darcy's equation. The spectral method is used to approximate its solution. The permeability coefficient depends on the pressure. We have proved that the discrete scheme converges to the solution of the continuous problem.

Furthermore, numerical tests confirm our theoretical results, in particular time and space accuracy in dimension 2 and 3. Finally, in the last test in paragraph 7.3, and in order to perform a somewhat realistic computation of the model we used, we correctly reproduce the simulation in dimension 3.

\section{References}

[1] Y. Achdou, C. Bernardi, and F. Coquel. A priori and a posteriori analysis of finite volume discretizations of Darcy's equations. Numer. Math., 96(1):17-42, 2003.

[2] R.A. Adams and J. Fournier. Sobolev spaces. Academic Press, 2003.

[3] E. Ahusborde, M. Azaïez, F. Ben Belgacem, and C. Bernardi. Automatic simplification of Darcy's equations with pressure dependent permeability. ESAIM Math. Model. Numer. Anal., 47(6):1797-1820, 2013.

[4] M. Azaïez, F. Ben Belgacem, C. Bernardi, and N. Chorfi. Spectral discretization of Darcy's equations with pressure dependent porosity. Appl. Math. Comput., 217:1838-1856, 2010.

[5] J.-M. Bernard. Density results in Sobolev spaces whose elements vanish on a part of the boundary. Chin. Ann. Math. Ser. B, 32(6):823-846, 2011.

[6] C. Bernardi, V. Girault, and K. Rajagopal. Discretization of an unsteady flow through porous solid modeled by Darcy's equations. Math. Models Methods Appl. Sci., 18(12):20872123, 2008.

[7] C. Bernardi and Y. Maday. Spectral methods. In Handbook of Numerical Analysis, Vol. V, 209 - 485. North-Holland, Amsterdam, 1997.

[8] C. Bernardi, Y. Maday, and F. Rapetti. Discrétisations variationnelles de problèmes aux limites elliptiques, volume 45 of Mathématiques \& Applications. Springer-Verlag, Paris, 2004. 
[9] H. Brezis. Analyse Fonctionnelle : Théorie et Applications. Collection "Mathématiques Appliquées pour la Maîtrise", Masson, 1983.

[10] H. Brezis and P. Mironescu. Gagliardo-Nirenberg, composition and products in fractional Sobolev spaces. J. Evol. Equ., 1(4):387-404, 2001. Dedicated to the memory of Tosio Kato.

[11] F. Brezzi, J. Rappaz, and P.-A. Raviart. Finite-dimensional approximation of nonlinear problems. I. Branches of nonsingular solutions. Numer. Math., 36(1):1-25, 1980/81.

[12] V. Girault and P.-A. Raviart. Finite Element Methods for Navier-Stokes Equations, Theory and Algorithms, volume 5 of Springer Series in Computational Mathematics. SpringerVerlag, Berlin, 1986.

[13] J.-L. Lions and E. Magenes. Problèmes aux limites non homogènes et applications. Vol. 1. Dunod, Paris, 1968.

[14] Y. Maday and E.M. Rønquist. Optimal error analysis of spectral methods with emphasis on non-constant coefficients and deformed geometries. Comput. Methods Appl. Mech. Engrg., 80:91-115, 1990.

[15] A. Quarteroni. Some results of Bernstein and Jackson type for polynomial approximation in $L^{p}$-spaces. Japan J. Appl. Math., 1(1):173-181, 1984.

[16] K. R. Rajagopal. On a hierarchy of approximate models for flows of incompressible fluids through porous solids. Math. Models Methods Appl. Sci., 17(2):215-252, 2007.

[17] Y. Saad. Iterative Methods for Sparse Linear Systems. SIAM, Philadelphia, 2003.

[18] D. Yakoubi. Analyse et mise en œuvre de nouveaux algorithmes en méthodes spectrales. PhD thesis, Université Pierre et Marie Curie, Paris, Décembre 2007. 